\title{
Transcription factor competition facilitates self-sustained oscillations in single gene genetic circuits
}

\author{
Jasper Landman, ${ }^{1,2,3}$ Sjoerd M. Verduyn Lunel, ${ }^{4}$ and Willem K. Kegel ${ }^{2, *}$ \\ ${ }^{1}$ Laboratory for Physics $\&$ Physical Chemistry of Foods, \\ Wageningen University \& Research, \\ 6708 PB Wageningen, the Netherlands \\ ${ }^{2}$ Van 't Hoff Laboratory for Physical \& Colloid Chemistry, \\ Utrecht University, 3584 CH Utrecht, the Netherlands \\ ${ }^{3}$ Laboratory for Physical Chemistry, \\ Eindhoven University of Technology, \\ 5612 AZ Eindhoven, the Netherlands \\ ${ }^{4}$ Mathematisch Instituut, Utrecht University, 3584 CD Utrecht, the Netherlands
}

(Dated: November 30, 2021) 


\begin{abstract}
Genetic feedback loops can be used by cells as a means to regulate internal processes or keep track of time. It is often thought that, for a genetic circuit to display self-sustained oscillations, a degree of cooperativity is needed in the binding and unbinding of actor species. This cooperativity is usually modeled using a Hill function, regardless of the actual promoter architecture. Moreover, genetic circuits do not operate in isolation and often transcription factors are shared between different promoters. In this work we show how mathematical modelling of genetic feedback loops can be facilitated with a mechanistic fold-change function that takes into account the titration effect caused by competing binding sites for transcription factors. The model shows how the titration effect aids self-sustained oscillations in a minimal genetic feedback loop: a gene that produces its own repressor directly — without cooperative transcription factor binding. The use of delaydifferential equations leads to a stability contour that predicts whether a genetic feedback loop will show self-sustained oscillations, even when taking the bursty nature of transcription into account.
\end{abstract}

\title{
I. INTRODUCTION
}

If the Nobel prize in chemistry of 2017 — given jointly to Michael Young, Michael Rosbash and Jeffrey Hall for their work on the molecular mechanisms controlling the circadian rhythm[1-3] — is any indication, it is that oscillating reactions in living cells are a hot topic. The cellular analogue of classical clock reactions like the Belousov-Zhabotinsky reaction, $[4,5]$ self-sustained oscillations require a network of reactions that lead to a negative feedback loop. [2, 6-16] This oscillating network can in turn be used to coordinate important processes in the cell, such as cell division.[8, 11, 13, 17-20] Oscillatory circuits may include both enzymatic binding and unbinding events, as well as transcriptional elements, although the majority of cellular oscillatory reactions that have been observed incorporate a transcription event in the feedback loop[21] and as such have the potential to regulate parallel transcription processes. Progress in synthetic biology has made it possible to design circuits of transcriptional elements that perform desired functions within a cell (see e.g. [22-25]-) or even across multiple organisms [26].

According to Novák and Tyson [21], sustained oscillations need the following ingredients:

\footnotetext{
*W.K.Kegel@uu.nl
} 
first of all, as was already indicated before, a negative feedback loop is necessary. Without a negative feedback loop a system can not be brought back to its original state. The second requirement is a delay in the feedback loop. When the feedback is instantaneous, any perturbation can be brought back immediately to its steady-state. And finally, a certain degree of cooperativity is required, without which sustained oscillations are not seen. In models, this cooperativity is usually modelled with a Hill function [27] in the binding probability of a transcription factor. The Hill function has the following form

$$
\theta=\frac{[\mathrm{L}]^{n}}{K_{\mathrm{d}}^{n}+[\mathrm{L}]^{n}}, \quad \text { (Hill function) }
$$

where $\theta$ is the occupation fraction of an adsorbed ligand to a lattice site, $[\mathrm{L}]$ is the concentration of ligand (monomer), $K_{\mathrm{d}}$ the dissociation constant and $n$ the Hill coefficient. For $n=1$ the Hill isotherm is equal to the Langmuir isotherm. The Hill isotherm was derived for the cooperative binding of oxygen to hemoglobin,[27] modelling it as the simultaneous binding of $n$ ligands to a lattice site. However, in many instances in literature, a Hill isotherm is used to describe the binding of a ligand with a different binding architecture. In those cases, an 'effective' Hill coefficient is used as a fitting parameter and a measure for the cooperativity of binding. The consequence of a higher effective Hill coefficient on the binding isotherm of a ligand is a very sharp transition from mostly free ligand to mostly bound ligand. It is this steep response that leads to oscillations.

Often, a transcription factor involved in an oscillatory circuit is shared by a number of other genes. The circuit and the regulated gene are effectively competing for a common pool of transcription factors, [28-30] which consequently leads to a titration effect - A dramatic increase in transcription factor occupancy is observed when the copy number of transcription factors crosses over from a regime where transcription factors are limiting to a regime of transcription factor excess. The increase is similar to the sharp transition found in the cooperative binding of ligands, as illustrated in fig. 1.

While the notion exists that this competition-driven titration effect can also lead to sufficient nonlinearity to generate self-sustained oscillating reactions — such circuits are coined 'titration oscillators'[31-33] - modelling of these circuits so far has resorted to the use of Hill isotherms with a phenomenological Hill coefficient.[34-38] Moreover, while the occupancy of transcription factor binding sites can be reasonably modelled for genes in isolation, the Hill function is at a disadvantage in a competitive environment, as we show 
FIG. 1. The trouble with Hill functions. a Fold-change of a gene regulated by a simple repression scenario as a function of repressor copy number $P$, in the presence and absence of competitor sites is captured accurately by the grand canonical formalism. Using Hill functions, the Langmuir-behaviour in the absence of competitors can be accurately described. However, the Hill function can not capture the full behaviour of the titration effect caused by competitor sites, even when fitting both the Hill coefficient and the binding free energy. The small mismatch between the grand canonical and Hill curves at low transition factor copy number is a known effect of the differences in statistical mechanical ensemble in which these curves were calculated.[28] b The occupancy $\theta$ of repressors to their binding sites is captured well by the Hill function. c The dramatic switching behaviour is a consequence of the lowered transcription factor fugacity at concentrations lower than the number of competitor sites. The binding free energy of repressors was kept at $-15 k_{\mathrm{B}} T$ and for competitor sites at $-18 k_{\mathrm{B}} T$. The number of non-specific sites was kept at $5 \times 10^{6}$. The Hill function in the presence of competitors was given an effective binding energy of $-11.3 k_{\mathrm{B}} T$ and Hill coefficient of 7 . The model used to calculate these curves was presented in Weinert et al. [28], Landman et al. [29].

in fig. 1 for a gene regulated by a simple repression architecture. Kim and Tyson [39] level similar criticism at the use of quasi-Steady State Michaelis Menten kinetics, the result of which reduces to a Hill isotherm.

In the absence of competitor sites, transcription factor binding follows the Langmuir isotherm. In the presence of 40 competitor sites, the sharp switching behaviour of the occupancy can be captured by the Hill function, yet both the Hill coefficient and the binding free energy have now become fitting parameters of obscure physical relevance, and still the resulting function does not capture the full fold-change behaviour.

In this article we will show how the rate equations that govern the behaviour of genetic circuits lead to a very natural inclusion of previously derived results.[28-30] Within the assumption that transcription and translation are slow processes in comparison to the binding and unbinding kinetics of transcription factors, we can use expressions for the fold-change of genes derived in the grand canonical ensemble, which account for the presence of other binding sites, such as multiple gene copies, competitor sites and inhibitors, competing for the same transcription factor, and for complex regulatory architectures with multiple bind- 
ing sites. We show that the presence of a small number of competitor sites or inhibitors within the cell generates sufficient nonlinearity in the response curve of the gene to allow self-sustained oscillation in a minimal genetic feedback loop: a gene that produces its own repressor.

We use delay differential equations (DDEs)[40] to represent the finite time taken by the transcriptional and translational process. DDEs allow us to relate the change in mRNA copy number to the concentration of transcription factors at some time in the past. We restrict ourselves to DDEs with constant time delays and do not include the stochastics of transcription and translation explicitly. For that, a master equation approach would be required. We show, however, that one can account for variances in the time delay implicitly to evaluate the stability of an oscillating circuit. Korsbo and Jönsson [41] are critical of the use of DDEs within the context of transcriptional regulation, and provide an alternative approach based on a constant rate of information propagation along a variable path length. While constant delay DDEs may not capture the full behaviour of this proposed alternative, the use of DDEs provides a much more realistic theory than can be given using ordinary differential equations, while allowing for a full stability and bifurcation analysis with delay as a bifurcation parameter. Such an analysis was until recently only possible for ordinary differential equations.[40] In the future we plan to extend our model to include stochastic perturbations explicitly. Finally, we show that for a single membered genetic circuit it is possible to derive a criterion that predicts whether self-sustained oscillations are possible. The criterion depends only on the slope of the fold-change curve of the promoter architecture, and the delay time that is inherent in the transcription and translation process.

\section{RESULTS \& DISCUSSION}

\section{A. Rate equations}

The change in the copy number of a protein $\mathrm{P}$ is given by two contributions. The first is the first order degradation of the protein by the cellular recycling machinery. The second is the synthesis of $\mathrm{P}$ by the ribosomes, the rate of which is proportional to the concentration of P-encoding mRNA (M). The rate equation describing the copy numbers $\mathbf{P}$ of a set of 
proteins involved in a genetic circuit is given by

$$
\frac{\mathrm{d} \mathbf{P}}{\mathrm{d} t}=-\Gamma_{\mathrm{P}} \mathbf{P}+k_{\mathrm{r}} \mathbf{M}
$$

with $\mathbf{P}, \mathbf{M}$ the copy numbers of protein and mRNA respectively. Furthermore, $\Gamma_{\mathrm{P}}$ and $k_{\mathrm{r}}$ are diagonal matrices containing the first order degradation rate constants, and the activity rate constants of the ribosomes. The mRNA is produced by RNA polymerase at the gene of interest at a rate that depends on the arrangement of transcription factors. For all proteins involved in the genetic circuit of interest, these rates are given by the vector $\mathbf{k}_{\mathbf{s}}$. Since transcription - the synthesis of mRNA - and the transport to the ribosomes takes a finite amount of time, we explicitly introduce a time delay $\tau$ to account for that delay. At the same time, mRNA also undergoes first order degradation by the cellular machinery with rate constants given by the diagonal matrix $\Gamma_{\mathrm{M}}$. The mRNA degradation rate constants are typically comparable in magnitude to the protein rate constants $\Gamma_{\mathrm{P}}$.[15] In fact, the dominant mechanism causing this first order decay is dilution due to a global growth rate $\mu$ although active degradation mechanisms may alter the individual degradation rate constants. The rate equation describing the change in mRNA copy numbers $\mathbf{M}$ is then given by

$$
\frac{\mathrm{d} \mathbf{M}}{\mathrm{d} t}=-\Gamma_{\mathrm{M}} \mathbf{M}+\mathbf{k}_{\mathrm{s}}(\mathbf{P}(t-\tau)) .
$$

This rate equation is formally a delay differential equation (DDE)[40], since it depends on the transcriptional activity of the genes $\mathbf{k}_{\mathrm{s}}$ at a time in the past. The rate constants $\mathbf{k}_{\mathrm{s}}$ depend nonlinearly on the concentration of transcription factors that influence the transcription of the gene. We take the individual transcription rates $k_{\mathrm{s}}^{i}$ as proportional to the (equilibrium) probability that their promoter region is occupied by RNA polymerase (RNAP), an assumption which is justified when the formation of the RNAP open complex on the promoter site of a gene is slow in comparison to the binding and unbinding kinetics of transcription factors over the genome [42-45]. Under these assumptions, equilibrium statistical mechanics can be used to calculate the RNAP occupancy. The assumptions needed to treat transcription regulation as a quasi-equilibrium process are subtle (see e.g. ref [46, 47], and there exists a corresponding class of kinetic models, which do not require as many assumptions, at the cost of requiring more parameters. [48-54] Within these assumptions, the rates of mRNA synthesis can be approximated as

$$
k_{\mathrm{s}}^{i}(t)=k_{0}^{i} N^{i} \theta^{i}(\mathbf{P}(t))
$$


where $k_{\mathrm{s}}^{i}$ are the elements of the vector $\mathbf{k}_{\mathrm{s}}$. Furthermore, $k_{0}^{i}$ is the (constant) rate at which mRNA of protein $i$ is produced when RNAP reads the promoter sequence, $N^{i}$ is the number of gene copies contributing to the production of the protein and $\theta^{i}$ the occupational fraction of the promoter, calculated as the fraction of all configurational microstates where the RNA polymerase occupies the promoter sequence. As such, the occupational fraction $\theta^{i}$ (and by extension, $k_{\mathrm{s}}^{i}$ ) is not a function of time directly, but rather a function of all transcription factor copy numbers $\mathbf{P}$ affecting this binding probability at a given time.

When the gene is completely unregulated, that is, in the absence of any regulatory proteins, the concentration of mRNA can reach a steady-state. In that case, we can write for each protein $i$ the steady-state equation

$$
\gamma_{\mathrm{M}}^{i} M^{i,(0)}=k_{0}^{i} N^{i} \theta^{i,(0)}
$$

Here, $M^{i,(0)}$ is the steady-state copy number of mRNA encoding protein $i$ in the absence of any regulation, and $\gamma_{\mathrm{M}}^{i}$ is the $i$ th diagonal element of matrix $\Gamma_{\mathrm{M}}$. Inserting eq. (5) into eq. (3), we obtain for the instantaneous (not steady-state) copy number of each mRNA species $i$

$$
\frac{\mathrm{d} M^{i}}{\mathrm{~d} t}=-\gamma_{\mathrm{M}}^{i} M^{i}+\gamma_{\mathrm{M}}^{i} M^{i,(0)} \frac{\theta^{i}(\mathbf{P}(t-\tau))}{\theta^{i,(0)}}
$$

The fraction $\theta^{i}(\mathbf{P}(t-\tau)) / \theta^{i,(0)}$ we recognise as the (instantaneous) fold-change. The (instantaneous) fold-change acts as an input-output function that depends on the architecture of the gene promoter sequence. Expressions for the fold-change that take into account the promoter architecture and the presence of competing binding sites for transcription factors can be derived in a straightforward manner using the grand canonical ensemble. [29].

Similarly, the rate of protein synthesis at the ribosomes in steady-state is given by

$$
\gamma_{\mathrm{P}}^{i} P^{i,(0)}=k_{\mathrm{r}}^{i} M^{i,(0)}
$$

with $P^{i}, P^{i,(0)}$ the actual and unregulated copy numbers of protein $i$ respectively, and $\gamma_{c e P}^{i}$ the $i$ th diagonal element of $\Gamma_{\mathrm{P}}$. From the steady-state equation we can obtain a measure for $k_{\mathrm{r}}^{i}$ in terms of the steady-state copy numbers of protein and mRNA.

It is convenient here to normalise the copy numbers of proteins and mRNA by their steady-state copy numbers. We therefore introduce the normalised copy numbers $\mathbf{p}, \mathbf{m}$ 
which are defined for each element in the vectors by $p^{i} \equiv P^{i} / P^{i,(0)}, m^{i} \equiv M^{i} / M^{i,(0)}$, so that we obtain the following system of DDEs.

$$
\left\{\begin{array}{l}
\frac{\mathrm{d} \mathbf{m}}{\mathrm{d} t}=-\Gamma_{\mathrm{M}} \mathbf{m}+\Gamma_{\mathrm{M}} \text { fold-change }(\mathbf{p}(t-\tau)) \\
\frac{\mathrm{d} \mathbf{p}}{\mathrm{d} t}=-\Gamma_{\mathrm{P}} \mathbf{p}+\Gamma_{\mathrm{P}} \mathbf{m}
\end{array}\right.
$$

There are three relevant cases between which we make a distinction. When a stable steady-state is reached, $p$ is determined only by the fold-change. Alternatively, when

$\Gamma_{\mathrm{M}} \gg \Gamma_{\mathrm{P}}$, the concentrations of mRNA quickly reach a steady-state and the system of rate equations reduces to

$$
\frac{\mathrm{d} \mathbf{p}}{\mathrm{d} t}=-\Gamma_{\mathrm{P}} \mathbf{p}+\Gamma_{\mathrm{P}} \text { fold-change }(\mathbf{p}(t-\tau)) . \quad\left(\Gamma_{\mathrm{M}} \gg \Gamma_{\mathrm{P}}\right)
$$

In the case that both degradation constants are comparable, the full system of equations eq. (8) should be used. The quantity called fold-change acts as the input-output function that replaces the Hill function used in many previous works, and its form depends on the regulatory architecture of interest. It remains dependent on the copy numbers of transcription factors that are involved, and it is through this that the dynamics of one gene can be coupled to a different gene.

\section{B. Single gene oscillator}

FIG. 2. The copy number titrating oscillator. a Protein (solid lines) and mRNA (dotdashed lines) copy number as function of time for a gene regulated by $\mathbf{b}$ simple repression scenario with $N$ gene copies, producing its own repressor. c Phase space trajectories of a, shown in conjunction with the nullclines. Because of the time delay in evaluating the magnitude of $\dot{m}$, the phase space trajectories do not cross the $\dot{m}$-nullcline completely horizontally. For a sufficiently high gene copy number a stable limit cycle is reached. $\mathbf{d}$ Fold-change of the gene as function of the total number of transcription factors.

The simplest feedback loop one can think of is a gene, regulated by a simple repression promoter architecture, that directly produces its own repressor, P. We show a schematic of the architecture in fig. 2(b). We previously derived the fold-change relation for this 
architecture, $[28,29]$ which in the weak promoter limit reads

$$
\text { fold-change }=\frac{1}{1+\lambda_{\mathrm{P}} \exp -\beta \epsilon_{\mathrm{P}}},
$$

where $\beta=\left(k_{\mathrm{B}} T\right)^{-1}, \epsilon_{\mathrm{P}}$ is the binding (free) energy of $\mathrm{P}$ to its specific binding site on the gene, and $\lambda_{\mathrm{P}}=\exp \beta \mu_{\mathrm{P}}$ is the transcription factor fugacity, which is found self-consistently from the total transcription factor copy number within the cell. The fugacity of repressors therefore depends on the number and binding energy of its specific binding sites, of any competitor genes that also bind the repressor, and to the reservoir of non-specific binding sites on the DNA. We assume that the fraction of transcription factors unbound from the DNA is negligible, and set the effective binding energy to the non-specific sites to 0.[55] The equation is valid for multiple independent copies of the same gene, competing for the same pool of transcription factors.

We numerically integrate eq. (8) for different gene copy numbers using the method of steps, starting with a protein and mRNA copy number of 0 over the time interval $[-\tau, 0]$. The transcription factor binding free energy was $-15 \mathrm{k}_{\mathrm{B}} \mathrm{T}$ for the specific sites. The steadystate unregulated repressor copy number $P^{(0)}$ was 5 per gene copy. For these trajectories, we did not include any other competitor genes. Finally, the protein and mRNA degradation rates $\gamma_{\mathrm{P}}, \gamma_{\mathrm{M}}$ were $0.03 \mathrm{~min}^{-1}$ and the delay time $\tau$ was $18.5 \mathrm{~min}$ as per ref [15]. The different trajectories are shown in fig. 2 .

FIG. 3. The single gene oscillator. a Protein (solid lines) and mRNA (dotdashed lines) copy number as function of time for a gene regulated by $\mathbf{b}$ simple repression scenario producing its own repressor, with $N_{\mathrm{c}}$ sites competing for a common pool of repressors. c Phase space trajectories of a, shown in conjunction with the nullclines. Because of the time delay in evaluating the magnitude of $\dot{m}$, the phase space trajectories do not cross the $\dot{m}$-nullcline completely horizontally. For a sufficiently high competitor copy number a stable limit cycle is reached. d Fold-change of the gene as function of the total number of transcription factors. Note that for the dynamic behaviour, it does not matter whether the competitor sites are DNA binding sites, enzymes or other ligands binding the repressors.

In fig. 2 we see that for a low copy number of genes, the concentrations of mRNA and repressor quickly proceed to a stable value, with an initial overshoot that is corrected for 
quickly. This can graphically be seen in the phase space figure in fig. 2(c). The phase space trajectory quickly spirals to the stable point at the intersection of the two nullclines. For higher gene copy numbers, we see first the same qualitative picture, although the oscillations dampen out at a lower rate. Above a certain threshold copy number, the oscillations become self-sustained and we see the phase space trajectory approach a stable limit cycle, centred around the intersection between the nullclines. These self-sustained oscillations only appear with a nonzero delay time $\tau$.

Self-sustained oscillations can also be attained with just a single gene copy, in the presence of a reservoir of competitor genes. We numerically integrate eq. (8) for a single gene copy in the presence of a number of competitor sites. We used a binding energy to the competitor sites of $-18 \mathrm{k}_{\mathrm{B}} \mathrm{T}$. In this case, we set the steady-state unregulated repressor copy number $P^{(0)}$ to 100 per gene copy. A high baseline activity is necessary here: without sufficient repressors in the cell, the gene will be almost completely outcompeted by the competitor sites. All other details were kept as before. The results of the numerical integration can be seen in fig. 3. The results are similar to the previous case: When there are multiple binding sites competing for the repressor, the response curve becomes steeper and self-sustained oscillations are attained.

FIG. 4. The looping gene oscillator. a Protein and mRNA copy number as function of time for a gene regulated by $\mathbf{b}$ a looping scenario producing its own repressor. $\mathbf{c}$ Phase space trajectories of a, shown in conjunction with the nullclines. Because of the time delay in evaluating the magnitude of $\dot{m}$, the phase space trajectories do not cross the $\dot{m}$-nullcline completely horizontally. For a sufficiently favourable looping free energy a stable limit cycle is reached. Here, the steady-state unregulated repressor copy number was kept at $P^{(0)}=5$. d Fold-change of the gene as function of the total number of transcription factors.

Finally, the promoter architecture itself can generate sufficient nonlinearity to sustain oscillations in a genetic feedback loop that only involves a single gene copy. In the looping architecture [56] transcription factors have two DNA binding domains that are capable of binding two operator sites simultaneously. An auxiliary operator site near the promoter can enhance the efficacy of the transcription factor by increasing the probability of occupancy of the main operator site, where it is able to regulate transcription, by allowing for loops in the DNA between the operator sites. In our previous work [29] we showed that the equation 
describing the fold-change in the grand canonical ensemble is given by

$$
\text { fold-change }=\frac{1+\lambda_{\mathrm{P}} x_{\mathrm{P}}^{\mathrm{a}}}{1+\lambda_{\mathrm{P}}\left(x_{\mathrm{P}}^{\mathrm{a}}+x_{\mathrm{P}}^{\mathrm{m}}+x_{\mathrm{P}}^{\mathrm{a}} x_{\mathrm{P}}^{\mathrm{m}} x_{\mathrm{L}}\right)+\lambda_{\mathrm{P}}^{2} x_{\mathrm{P}}^{\mathrm{m}} x_{\mathrm{P}}^{\mathrm{a}}} .
$$

Here, $x_{\mathrm{P}}^{\mathrm{m}, \mathrm{a}}=\exp -\beta \epsilon_{\mathrm{R}}^{m, a}$ are the Boltzmann factors of the binding free energies of the main (m) and auxiliary (a) operator sites, and $x_{\mathrm{L}}=\exp -\beta \Delta F_{\mathrm{L}}$ is the Boltzmann factor of the free energy of forming a transcription factor-DNA loop connecting the auxiliary and main operator sites. As before, $\lambda_{\mathrm{P}}=\exp \beta \mu_{\mathrm{P}}$ is the repressor fugacity that is found selfconsistently by applying mass conservation.

Forming a DNA loop between two bound sites on a transcription factor causes the DNA to adopt an entropically unfavourable conformation. The free energy penalty $\Delta F_{\mathrm{L}}$ associated with this loop depends on the length of the DNA included within the loop.[57] We numerically integrate the rate eq. (8) in fig. 4 for different values of $\Delta F_{\mathrm{L}}$, ranging between $7 \mathrm{k}_{\mathrm{B}} \mathrm{T}$ and $11 \mathrm{k}_{\mathrm{B}} \mathrm{T}$, starting with protein and mRNA copy numbers of 0 . As before, the transcription factor binding free energy was $-15 \mathrm{k}_{\mathrm{B}} \mathrm{T}$ for both the main and auxiliary operator site, and the steady state unregulated repressor copy number $P^{(0)}$ was 5 per gene copy. We observe self-sustained oscillations for the two lower looping free energies, with a very similar oscillation period. When the looping free energy is too high, the statistical weight of the looped configuration lowers and a stable steady state is observed instead.

\section{Stability analysis}

An important question is whether it is possible to predict, a priori, whether a given genetic feedback circuit will lead to self-sustained oscillations. This is of particular importance since transcription is inherently stochastic and especially fluctuations in delay time lead to irregular availability of mRNA. The traditional approach to evaluate the stability of stationary points in systems of ordinary differential equations (ODEs) [58] can be extended to DDEs, following the approach of Hale and Verduyn Lunel [40]. In the context of genetic circuits, we will follow their approach to analyse a generalised model for $n$ genes that interact through a common set of transcription factors. We will explicitly allow fluctuations in the delay times. In the system of equations from eq. (8), we now explicitly introduce time delays both in the transcription $\left(\tau_{\mathrm{P}}\right)$ as well as in the translation $\left(\tau_{\mathrm{M}}\right)$ steps. The model 
then becomes

$$
\left\{\begin{array}{l}
\dot{\mathbf{m}}=-\Gamma_{\mathrm{M}} \mathbf{m}+\Gamma_{\mathrm{M}} \text { fold-change }\left(\mathbf{p}\left(t-\tau_{\mathrm{P}}\right)\right) \\
\dot{\mathbf{p}}=-\Gamma_{\mathrm{P}} \mathbf{p}+\Gamma_{\mathrm{P}} \mathbf{m}\left(t-\tau_{\mathrm{M}}\right)
\end{array}\right.
$$

where as before, $\mathbf{m}, \mathbf{p}$ are vectors built up from $m^{i}, p^{i}$, the delays $\tau_{\mathrm{M}}, \tau_{\mathrm{P}}$ are vectors built up from the independent delay times $\tau_{\mathrm{M}}^{i}, \tau_{\mathrm{P}}^{i}$, and $\Gamma_{\mathrm{M}}, \Gamma_{\mathrm{P}}$ are diagonal matrices with elements $\gamma_{\mathrm{M}}^{i}, \gamma_{\mathrm{P}}^{i}$, for each gene $i$. The fold-change for each gene depends on the architecture. Keeping the model as general as possible, we consider it in principle a function of all proteins in p. Say that we found a stationary point at the intersection of the nullclines at $\left(\mathbf{m}^{*}, \mathbf{p}^{*}\right)$. In order to investigate the stability of this point, we will linearise the differential equations around the stationary point and find trajectories in the neighbourhood of the stationary point, in the form of $\mathbf{v} e^{\lambda t}$. These trajectories move exponentially outward or into the stable point, with a direction $\mathbf{v}$ which is to be determined later. We linearise the fold-change term in the equation for $\dot{\mathbf{m}}$ around $\left(\mathbf{m}^{*}, \mathbf{p}^{*}\right)$, which gives us the $n \times n$ matrix

$$
J_{m}=\Gamma_{\mathrm{M}} \frac{\mathrm{d}}{\mathrm{d} \mathbf{p}} \text { fold-change }(\mathbf{p}),
$$

evaluated at $\left(\mathbf{m}^{*}, \mathbf{p}^{*}\right)$. To find trajectories in the neighbourhood of the stationary point that behave as $\mathbf{v} e^{\lambda t}$, we make the substitutions $\mathbf{m}(t)=\mathbf{a} e^{\lambda t}, \mathbf{p}=\mathbf{b} e^{\lambda t}$, with $\lambda$ a complex scalar. This allows us to write eq. (12) in the form of a linear matrix equation.

$$
\lambda\left(\begin{array}{l}
\mathbf{a} \\
\mathbf{b}
\end{array}\right) e^{\lambda t}=\underbrace{\left(\begin{array}{cc}
-\Gamma_{\mathrm{M}} & J_{m} e^{-\lambda \tau_{\mathrm{P}}} \\
\Gamma_{\mathrm{P}} e^{-\lambda \tau_{\mathrm{M}}} & -\Gamma_{\mathrm{P}}
\end{array}\right)}_{\mathbf{A}}\left(\begin{array}{l}
\mathbf{a} \\
\mathbf{b}
\end{array}\right) e^{\lambda t}
$$

Here, $e^{-\lambda \tau_{\mathrm{P}}}, e^{-\lambda \tau_{\mathrm{M}}}$ are diagonal matrices consisting of elements $e^{-\lambda \tau_{\mathrm{P}, i}}, e^{-\lambda \tau_{\mathrm{M}, i}}$ for each gene $i$. The factors $e^{\lambda t}$ cancel out. Thus, we need to find eigenvalues $\lambda$ of the $2 n \times 2 n$ matrix $\mathbf{A}$. These eigenvalues can be found by solving the characteristic equation $\operatorname{det} \mathbf{A}-\lambda \mathbf{I}=0$, with I the identity matrix.

$$
\operatorname{det}\left(\begin{array}{cc}
-\Gamma_{\mathrm{M}}-\lambda \mathbf{I} & J_{m} e^{-\lambda \tau_{\mathrm{P}}} \\
\Gamma_{\mathrm{P}} e^{-\lambda \tau_{\mathrm{M}}} & -\Gamma_{\mathrm{P}}-\lambda \mathbf{I}
\end{array}\right)=0
$$

The roots of this equation in the complex plane determine the stability of the point $\left(\mathbf{m}^{*}, \mathbf{p}^{*}\right)$. If all the roots have negative real parts, then all exponential trajectories around the stationary point move inward and the stationary point is asymptotically stable. There are finitely many dominant roots, i.e., roots with the same real part such the real parts of all other 
roots are strictly less than the real part of the dominant roots. The stability contour can be traced by logging when dominant roots cross the imaginary axis in the complex plane. If a dominant root crosses the imaginary axis with nonzero imaginary part, i.e., the real part of a complex dominant root crosses 0 , a Hopf bifurcation usually occurs and oscillatory behaviour is seen.

For a circuit with a single gene in a negative feedback loop, eq. (15) simplifies to

$$
\frac{\left(\lambda+\gamma_{\mathrm{M}}\right)\left(\lambda+\gamma_{\mathrm{P}}\right)}{\gamma_{\mathrm{M}} \gamma_{\mathrm{P}}} e^{\lambda \tau}-\frac{\mathrm{d}}{\mathrm{d} p} \text { fold-change }\left(p^{*}\right)=0,
$$

with $\tau=\tau_{\mathrm{M}}+\tau_{\mathrm{P}}$ the total delay time, a confirmation that only the total delay time in the feedback loop determines the local stability. We can make the equation dimensionless by rescaling the time by $\gamma \equiv \sqrt{\gamma_{\mathrm{M}} \gamma_{\mathrm{P}}}$. We introduce the rescaled degradation constants $\gamma_{m}, \gamma_{p}$, and redefine $\lambda$ as the rescaled characteristic value, resulting in the equation

$$
\left(\lambda+\gamma_{m}\right)\left(\lambda+\gamma_{p}\right) e^{\lambda \gamma \tau}-\frac{\mathrm{d}}{\mathrm{d} p} \text { fold-change }\left(p^{*}\right)=0 .
$$

FIG. 5. Stability contours for a single gene oscillator. a Stable oscillations are expected when the dominant roots of eq. (17) have a positive real part. The dotted line gives the contour where the real part of the dominant roots of eq. (17) is 0 . The colours indicate the imaginary part of the positive dominant root $\lambda$, which is related to the oscillation period. $\mathbf{b}$ From the Hopf bifurcation theorem, it follows that in first order approximation, the period of the oscillation is given by $2 \pi$ divided by the imaginary part of the dominant roots $\lambda$ at the stability contour. The symbols denote the period of a number of numerical integrations for different $P^{(0)}$. The y-axis is given in units of dimensionless time $\gamma t$. c,d,e Slope of the fold-change in the stationary point for $\mathbf{c} N$ copies, $\mathbf{d}$ a single copy in the presence of $N_{\mathrm{c}}$ competitor sites and $\mathbf{e}$ a single copy with a Looping architecture in the absence of competitor sites, of a gene regulated by its own product. Only when the slope in the stationary point crosses the threshold given by the stability contour in a are self-sustained oscillations possible. The grey region denotes the extent the stability contour shifts when a $20 \%$ variation is present in $\gamma \tau$.

In fig. 5 we plot the regions where self-sustained oscillations are possible and where the stationary point leads to a stable steady-state. The line along which the real part of the dominant eigenvalues $\lambda$ equal 0 - separating the two regions - is referred to as 
the stability contour, and was calculated by numerically solving eq. (17). The stability contour depends only on the rescaled delay time $\gamma \tau$ and the slope of the fold-change in the stationary point. We have taken $\gamma_{\mathrm{M}}=\gamma_{\mathrm{P}}=\gamma$ in this graph for convenience, but the figure does not significantly change when the two degradation constants are of comparable magnitude. When the delay is comparatively small, stable oscillations can only occur if the slope of the fold-change with respect to the normalised transcription factor copy number is very steep, corresponding to strong cooperativity or competition. At much larger delays, this requirement is less strict, although the slope should be steeper than -1 . As a consequence, a gene without a cooperative architecture will be unable to sustain oscillatory behaviour in isolation.[59]

Close around the stationary point, the system oscillates with a period of $2 \pi / \operatorname{Im}\{\lambda\}$. We show the positive branch of the real part of $\lambda$ in fig. 5a. However, since there are finitely many dominant roots, stable limit cycles do not oscillate infinitesimally close around the stationary point in phase space. Due to the Hopf bifurcation theorem, the oscillation occurs around the points in phase space where the real part of the dominant eigenvalue equals 0 , that is, where the oscillation does not grow or contract. As such, a better prediction of the period of a self-sustained oscillation is given by the $2 \pi$ over the imaginary part of the dominant eigenvalue along the stability contour, as we plot in fig. $5 \mathbf{b}$, together with the oscillation period obtained from numerical integrations of eq. (8). We see that indeed the oscillation period is always equal to or slightly larger than $2 \pi / \operatorname{Im}\{\lambda\}$.

Fluctuations in $\gamma \tau$ shift the stability contour locally: a system with a slope in the stationary point on the oscillation side of the stability contour could cross the boundary and become locally attractive. The oscillation of the system is expected to be erratic in such cases and lose its periodicity. On average, the stationary point is unstable, and the system tends away from it, but on short timescales the system may collapse back on the stationary point. The magnitude of the fluctuations in $\gamma \tau$ translate to a range of slopes for which this erratic behaviour is expected. In fig. $5 \mathbf{c}, \mathbf{d}, \mathbf{e}$ we have plotted the slope in the stationary point of the copy number titrating oscillator (c), the single gene oscillator $(\mathbf{d})$ and the looping oscillator (e), as a function of the steady state unregulated copy number of repressors per gene copy $P^{(0)}$. The grey dotted line denotes the stability contour for $\gamma \tau=0.555$ as per ref [15], with the grey region below denoting the shift in the stability contour upon a local $20 \%$ fluctuation in $\gamma \tau$ as a guess for the amount of fluctuations present in vivo. The 
representation in fig. $5 \mathbf{c}, \mathbf{d}, \mathbf{e}$ can show if self-sustained oscillations are possible for a given architecture, and what tolerance the system has towards variances in $\gamma \tau$ and $P^{(0)}$ — varying $P^{(0)}$ shifts the location of the stationary point, and thereby the slope of the fold-change curve in the stationary point. We can see in $\mathbf{c}$ that for the conditions in fig. 2 , the system only barely crosses the stability contour for $N=1000$ and would show erratic behaviour when fluctuations of $20 \%$ are present. However, for higher $P^{(0)}$, a very wide range of self-sustained oscillations are possible for $N=1000$. For gene copy numbers of $N \leq 100$ the slope in the stationary point does not cross the stability contour for any $P^{(0)}$, and stable steady states are expected. Similarly, in $\mathbf{d}$, we see that a single gene in the presence of 30 competitors can show self-sustained oscillations over a range of different $P^{(0)}$, while 20 competitors do not generate sufficient nonlinearity to cross the stability contour for any $P^{(0)}$. We note that the exact threshold will depend on mechanistic details such as the binding free energies of the gene and its competitor sites.

\section{CONCLUSIONS}

In this article we have shown that uncorrelated transcription factor binding can lead to self-sustained oscillations, even in a genetic feedback loop that only consists of a gene regulated by a simple repression architecture that directly produces its own repressor. The only condition for this to happen is that multiple transcription factor binding sites are coupled through their competition for a shared pool of transcription factors.

Using a mechanistic model for the fold-change of a promoter architecture[28, 29], the response of a gene to an external concentration of transcription factors could accurately be calculated in the competing limit. Importantly, the response functions follow directly from the regulatory architecture and are suitable for situations where transcription factors are shared by different promoter sites. Consequently, we no longer need to model the response of a gene with a phenomenological Hill function when the binding architecture demands a different isotherm.

We see that when a transcription factor is strongly competed for, the fold-change function of a gene that is regulated by that transcription factor becomes very sharp, even when binding is uncorrelated, that is, governed by a Langmuir isotherm. This titration effect, caused by competition, is able to provide a sufficiently steep response function for even 
minimal genetic feedback loops, where the gene produces its own repressor directly, to achieve self-sustained oscillations. Even though the individual gene copies are uncorrelated, they synchronise because they are coupled by a common transcription factor pool.

The main strength of our approach is the inclusion of a mechanistic gene response function within a relatively simple mathematical framework — delay differential equations — which allows us to derive a powerful stability criterion that can tell a priori whether a genetic feedback loop can show self-sustained oscillations. While DDEs do not explicitly include the fluctuations inherent in the stochastic transcription and translation processes, their use predicts the existence of an erratic regime arising from the fluctuations in transcriptional delay time. The extent by which the stability contour is crossed provides an estimation how robust the oscillations are with respect to stochastic noise in the transcription and translation process. Finally, it follows from the Hopf bifurcation theorem that the stability contour also provides a first order approximation to the oscillation period of the feedback loop.

[1] Nobelprize.org, The Nobel Prize in Physiology or Medicine 2017 (2017).

[2] P. E. Hardin, J. C. Hall, and M. Rosbash, Feedback of the Drosophila period gene product on circadian cycling of its messenger RNA levels., Nature 343, 536 (1990).

[3] M. W. Young and S. A. Kay, Time zones: a comparative genetics of circadian clocks, Nature Reviews Genetics 2, 702 (2001).

[4] A. M. Zhabotinsky, Periodical oxidation of malonic acid in solution (a study of the Belousov reaction kinetics), Biofizika 9, 306 (1964).

[5] B. P. Belousov, An oscillating reaction and its mechanism, Collection of Abstracts on Radiation Medicine , 145 (1959).

[6] K. Pye and B. Chance, Sustained sinusoidal oscillations of reduced pyridine nucleotide in a cell-free extract of Saccharomyces carlsbergensis., Proceedings of the National Academy of Sciences 55, 888 (1966).

[7] I. Prigogine, R. Lefever, A. Goldbeter, and M. Herschkowitz-Kaufman, Symmetry Breaking Instabilities in Biological Systems, Nature 223, 913 (1969).

[8] B. Hess and A. Boiteux, Oscillatory Phenomena in Biochemistry, Annual Review of Biochem- 
istry 40, 237 (1971).

[9] G. Gerisch, H. Fromm, A. Huesgen, and U. Wick, Control of cell-contact sites by cyclic AMP pulses in differentiating Dictyostelium cells, Nature 255, 547 (1975).

[10] L. F. Olsen and H. Degn, Chaos in an enzyme reaction, Nature 267, 177 (1977).

[11] T. Evans, E. T. Rosenthal, J. Youngblom, D. Distel, and T. Hunt, Cyclin: A protein specified by maternal mRNA in sea urchin eggs that is destroyed at each cleavage division, Cell 33, 389 (1983).

[12] J. Gerhardt, M. Wu, and M. W. Kirschner, Cell cycle dynamics of an M-phase specific cytoplasmic factor in Xenopus laevis oocytes and eggs, Journal of Cell Biology 98, 1247 (1984).

[13] J. C. Dunlap, Molecular Bases for Circadian Clocks, Cell 96, 271 (1999).

[14] M. B. Elowitz and S. Leibler, A synthetic oscillatory network of transcriptional regulators., Nature 403, 335 (2000).

[15] N. A. M. Monk, Oscillatory Expression of Hes1, p53, and NF-\$ \kappa \$B Driven by Transcriptional Time Delays, Current Biology 13, 1409 (2003).

[16] M. Gallego and D. M. Virshup, Post-translational modifications regulate the ticking of the circadian clock, Nature Reviews Molecular Cell Biology 8, 139 (2007).

[17] J. J. Tyson and B. Novák, Regulation of the Eukaryotic Cell Cycle: Molecular Antagonism, Hysteresis, and Irreversible Transitions, Journal of Theoretical Biology 210, 249 (2001).

[18] B. Novák and J. J. Tyson, Modeling the Cell Division Cycle: M-phase Trigger, Oscillations, and Size Control, Journal of Theoretical Biology 165, 101 (1993).

[19] Q. Yang and J. E. Ferrell, The Cdk1-APC/C cell cycle oscillator circuit functions as a timedelayed, ultrasensitive switch, Nature Cell Biology 15, 519 (2013).

[20] B. Novak and J. J. Tyson, Numerical analysis of a comprehensive model of M-phase control in Xenopus oocyte extracts and intact embryos., Journal of Cell Science 106, 1153 (1993).

[21] B. Novák and J. J. Tyson, Design principles of biochemical oscillators, Nature Reviews Molecular Cell Biology 9, 981 (2008).

[22] M. Xie and M. Fussenegger, Designing cell function: assembly of synthetic gene circuits for cell biology applications, Nature Reviews Molecular Cell Biology 19, 507 (2018).

[23] E. C. Greenwald, S. Mehta, and J. Zhang, Genetically Encoded Fluorescent Biosensors Illuminate the Spatiotemporal Regulation of Signaling Networks, Chemical Reviews 118, 11707 (2018). 
[24] P.-F. Xia, H. Ling, J. L. Foo, and M. W. Chang, Synthetic genetic circuits for programmable biological functionalities, Biotechnology Advances 37, 107393 (2019).

[25] J. Jaeger and N. Monk, Dynamical modules in metabolism, cell and developmental biology, Interface Focus 11, rsfs.2021.0011 (2021).

[26] Y. Chen, J. K. Kim, A. J. Hirning, K. Josić, and M. R. Bennett, Emergent genetic oscillations in a synthetic microbial consortium, Science 349, 986 (2015).

[27] A. V. Hill, The possible effects of the aggregation of the molecule of hemoglobin on its dissociation curves., Journal of Physiology 40, 4 (1910).

[28] F. M. Weinert, R. C. Brewster, M. Rydenfelt, R. Phillips, and W. K. Kegel, Scaling of Gene Expression with Transcription-Factor Fugacity, Physical Review Letters 113, 258101 (2014).

[29] J. Landman, R. C. Brewster, F. M. Weinert, R. Phillips, and W. K. Kegel, Self-consistent theory of transcriptional control in complex regulatory architectures, PLOS ONE 12, e0179235 (2017).

[30] R. C. Brewster, F. M. Weinert, H. G. Garcia, D. Song, M. Rydenfelt, and R. Phillips, The Transcription Factor Titration Effect Dictates Level of Gene Expression, Cell 156, 1 (2014).

[31] N. E. Buchler and M. Louis, Molecular Titration and Ultrasensitivity in Regulatory Networks, Journal of Molecular Biology 384, 1106 (2008).

[32] S. Karapetyan and N. E. Buchler, Role of DNA binding sites and slow unbinding kinetics in titration-based oscillators, Physical Review E 92, 062712 (2015).

[33] J. K. Kim and D. B. Forger, A mechanism for robust circadian timekeeping via stoichiometric balance, Molecular Systems Biology 8, 630 (2012).

[34] R. Lev Bar-Or, R. Maya, L. A. Segel, U. Alon, A. J. Levine, and M. Oren, Generation of oscillations by the p53-Mdm2 feedback loop: A theoretical and experimental study, Proceedings of the National Academy of Sciences 97, 11250 (2000).

[35] L. Glass and M. C. Mackey, Pathological conditions resulting from instabilities in physiological control systems, Annals of the New York Academy of Sciences 316, 214 (1979).

[36] H. Momiji and N. A. M. Monk, Dissecting the dynamics of the Hes1 genetic oscillator, Journal of Theoretical Biology 254, 784 (2008).

[37] L. Ma, J. Wagner, J. J. Rice, W. Hu, A. J. Levine, and G. A. Stolovitzky, A plausible model for the digital response of p53 to DNA damage, Proceedings of the National Academy of Sciences 102, 14266 (2005). 
[38] T. Zhang, P. Brazhnik, and J. J. Tyson, Exploring mechanisms of the DNA-damage response: p53 pulses and their possible relevance to apoptosis, Cell Cycle 6, 85 (2007).

[39] J. K. Kim and J. J. Tyson, Misuse of the Michaelis-Menten rate law for protein interaction networks and its remedy, PLOS Computational Biology 16, e1008258 (2020).

[40] J. K. Hale and S. M. Verduyn Lunel, Introduction to Functional Differential Equations, 1st ed. (Springer, New York, NY, 1993).

[41] N. Korsbo and H. Jönsson, It's about time: Analysing simplifying assumptions for modelling multi-step pathways in systems biology, PLOS Computational Biology 16, e1007982 (2020).

[42] D. K. Hawley and W. R. McClure, Mechanism of activation of transcription initiation from the lambda PRM promoter, Journal of Molecular Biology 157, 493 (1982).

[43] H. Buc and W. R. McClure, Kinetics of open complex formation between \textit\{Escherichia coli\} RNA polymerase and the \textit\{lac\} UV5 promoter. Evidence for a sequential mechanism involving three steps, Biochemistry 24, 2712 (1985).

[44] N. Mitarai, I. B. Dodd, M. T. Crooks, and K. Sneppen, The Generation of Promoter-Mediated Transcriptional Noise in Bacteria, PLoS Computational Biology 4, e1000109 (2008).

[45] N. Mitarai, S. Semsey, and K. Sneppen, Dynamic competition between transcription initiation and repression: Role of nonequilibrium steps in cell-to-cell heterogeneity, Physical Review E 92, 022710 (2015).

[46] J. Elf, G. W. Li, and X. S. Xie, Probing transcription factor dynamics at the single-molecule level in a living cell, Science 316, 1191 (2007).

[47] A. Sanchez, M. L. Osborne, L. J. Friedman, J. Kondev, and J. Gelles, Mechanism of transcriptional repression at a bacterial promoter by analysis of single molecules., The EMBO Journal 30, 3940 (2011).

[48] M. S. H. Ko, A stochastic model for gene induction, Journal of Theoretical Biology 153, 181 (1991).

[49] J. Peccoud and B. Ycart, Markovian modelig of gene product synthesis, Theoretical Population Biology 48, 222 (1995).

[50] M. T. Record Jr., W. S. Reznikoff, M. L. Craig, K. L. McQuade, and P. J. Schlax, Escherichia coli $\{$ RNA $\}$ polymerase (sigma70) promoters and the kinetics of the steps of transcription initiation, in In Escherichia coli and Salmonella Cellular and Molecular Biology, edited by N. F. C. e. al. (ASM Press, Washington DC, 1996) pp. 792-821. 
[51] T. B. Kepler and T. C. Elston, Stochasticity in transcriptional regulation: origins, consequences, and mathematical representations., Biophysical Journal 81, 3116 (2001).

[52] A. Sanchez and J. Kondev, Transcriptional control of noise in gene expression, Proceedings of the National Academy of Sciences 105, 5081 (2008).

[53] D. Michel, How transcription factors can adjust the gene expression floodgates, Progress in Biophysics \\& Molecular Biology 102, 16 (2010).

[54] R. Phillips, Napoleon Is in Equilibrium, Annual Review of Condensed Matter Physics 6, 85 (2015).

[55] J. Landman, R. N. Georgiev, M. Rydenfelt, and W. K. Kegel, In vivo and in vitro consistency of thermodynamic models for transcription regulation, Physical Review Research 10.1103/physrevresearch.1.033094 (2019).

[56] H. Salgado, M. Peralta-Gil, S. Gama-Castro, A. Santos-Zavaleta, L. Muñiz-Rascado, J. S. García-Sotelo, V. Weiss, H. Solano-Lira, I. Martínez-Flores, A. Medina-Rivera, G. SalgadoOsorio, S. Alquicira-Hernández, K. Alquicira-Hernández, A. López-Fuentes, L. Porrón-Sotelo, A. M. Huerta, C. Bonavides-Martínez, Y. I. Balderas-Martínez, L. Pannier, M. Olvera, A. Labastida, V. Jiménez-Jacinto, L. Vega-Alvarado, V. del Moral-Chávez, A. HernándezAlvarez, E. Morett, and J. Collado-Vides, RegulonDB v8.0: omics data sets, evolutionary conservation, regulatory phrases, cross-validated gold standards and more, Nucleic Acids Research 41, D203 (2013).

[57] J. Q. Boedicker, H. G. Garcia, and R. Phillips, Theoretical and Experimental Dissection of DNA Loop-Mediated Repression, Physical Review Letters 110, 018101 (2013).

[58] S. Strogatz, Non-linear Dynamics and Chaos, 1st ed. (Perseus Books, New York, 1994).

[59] The slope of the fold-change curve for a gene with an effective Hill coefficient of 1 never exceeds -1 with respect to the normalised repressor copy number. 
(a)

(b)

hioRxiv preprint doi: https://doi.org/10.1101/2021.12.01.470700; this version posted December 1, 2021. The copyright holder for this preprint

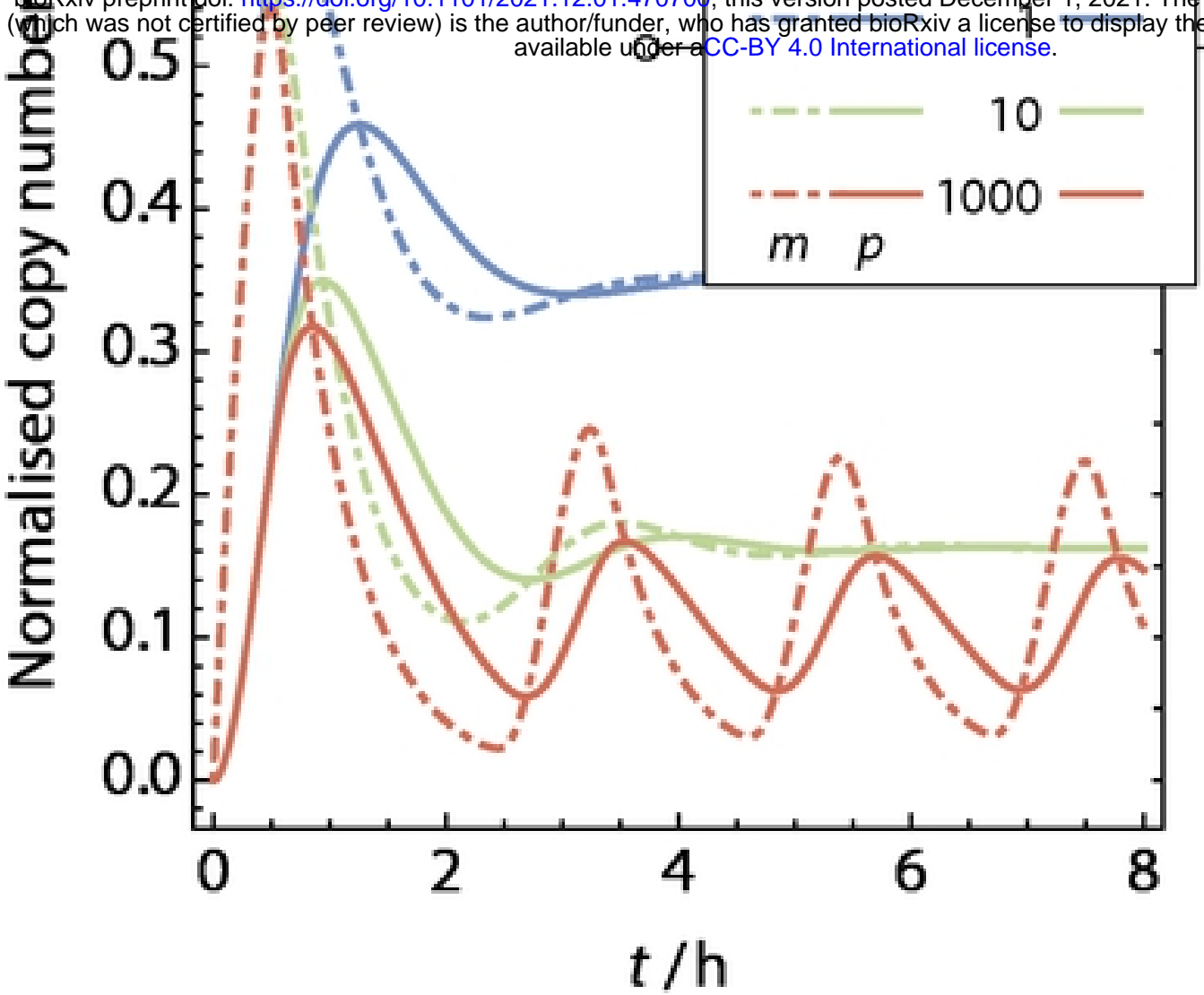

(c)

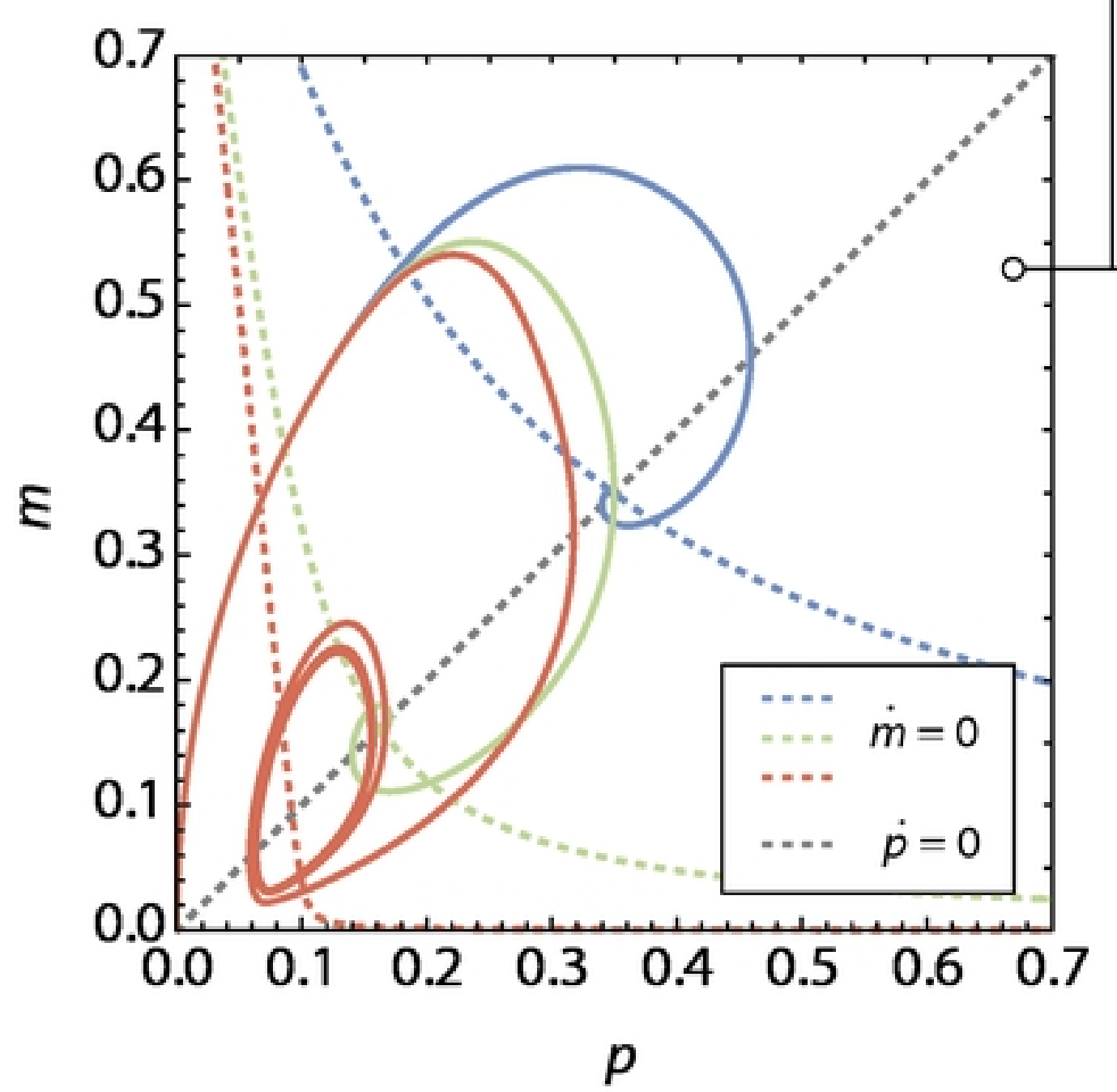

(d)

repressor binding site overlapping the promoter
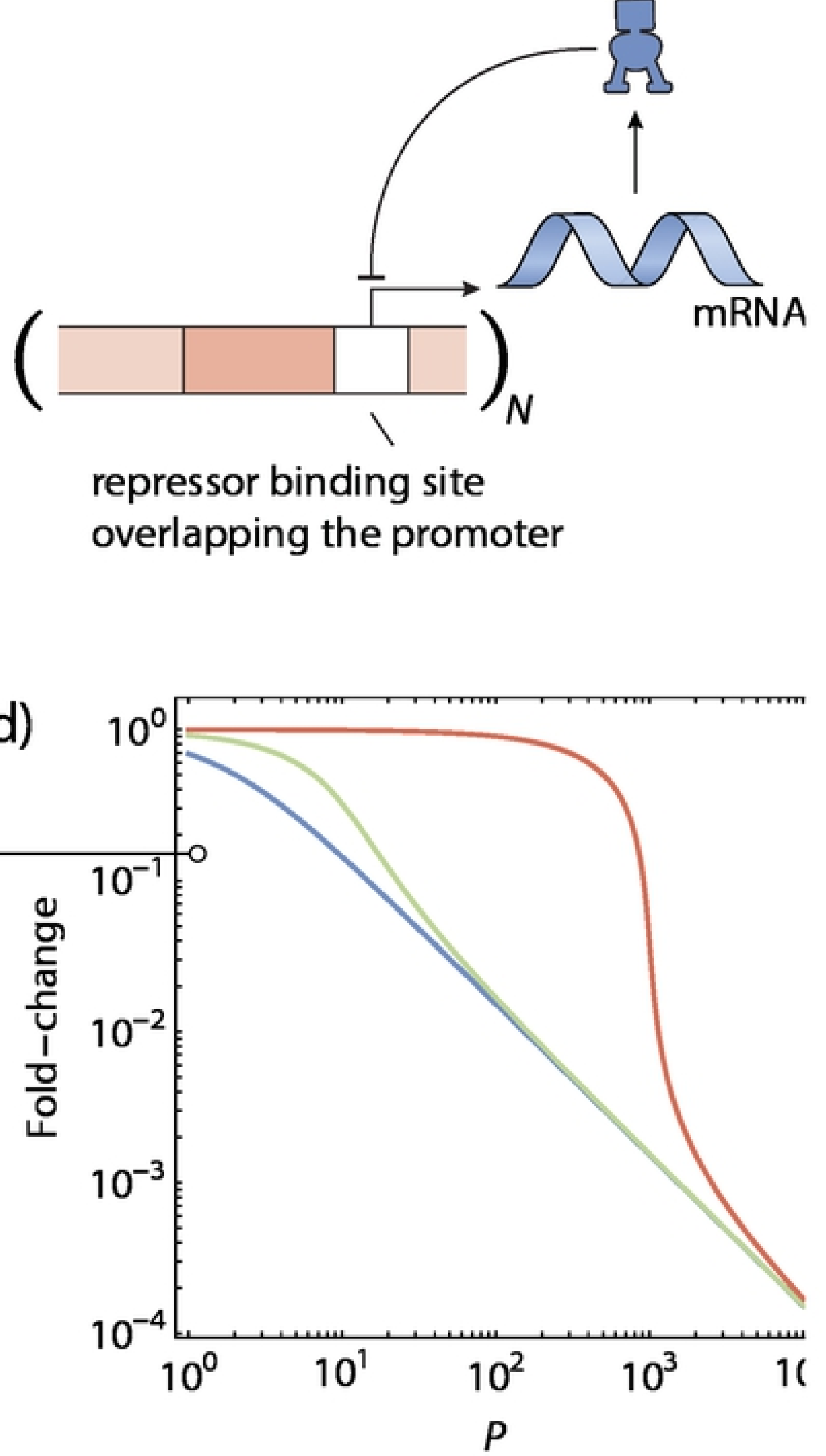

Figure 2 
(a)

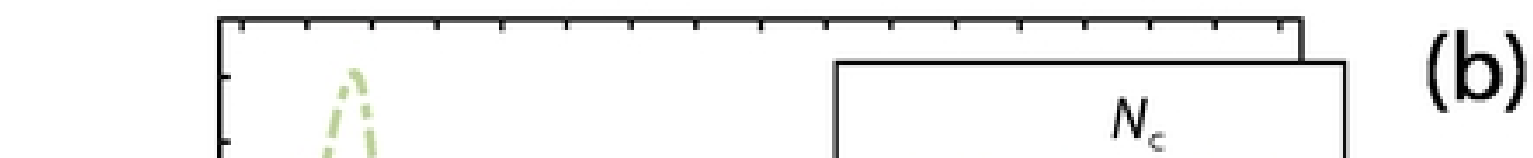

hioRxiv preprint doi: https://doi.org/10.1101/2021.12.01.470700; this version posted December 1, 2021. The copyright holder for this preprint
(vohich was not certified by peer review) is the author/funder, who has granted bioRxiv a liceose to display the preprint in perpetuity. It is made Q 0.6 - available undeCAce $B Y 4.0$ International license.

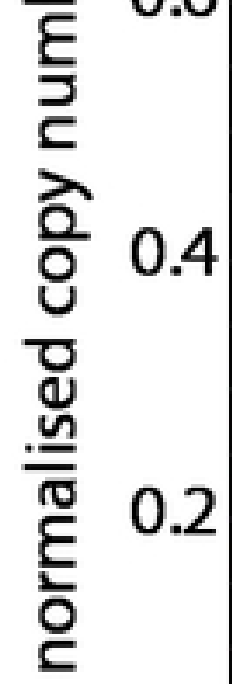
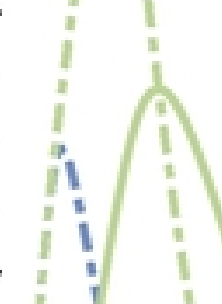

în
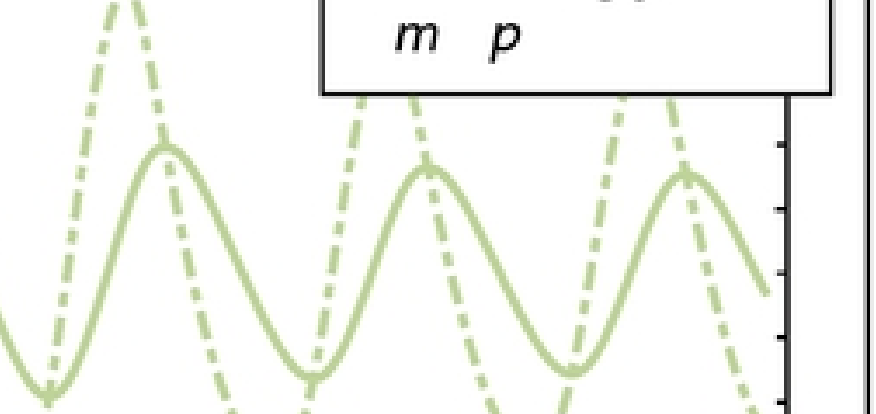
0.0
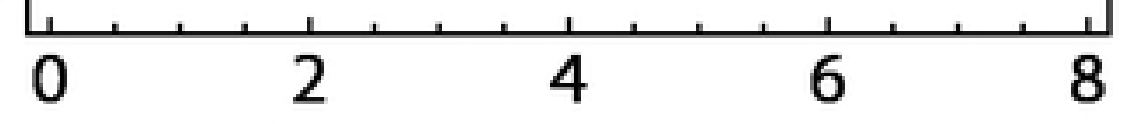

$t / \mathrm{h}$

(c)

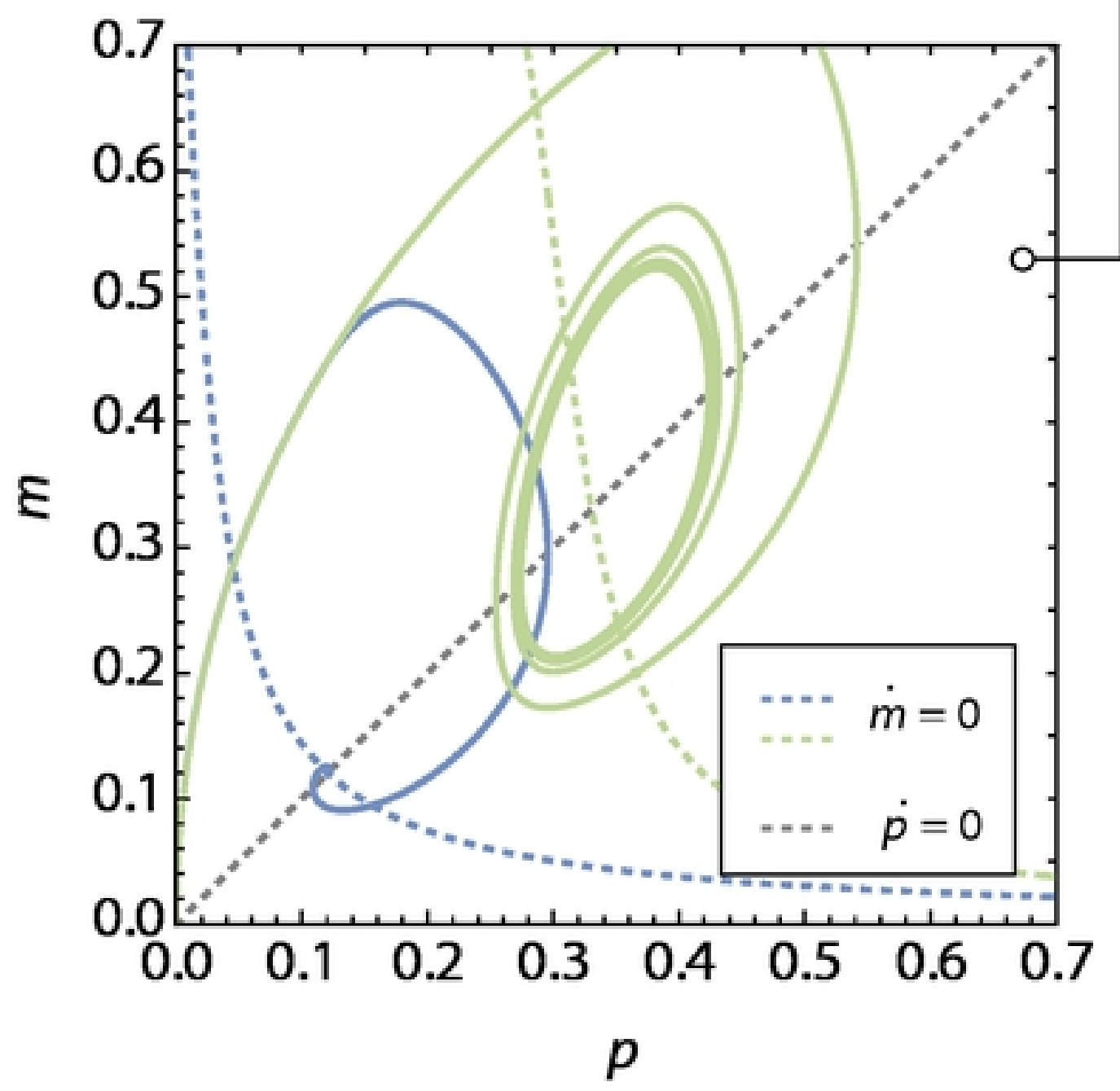

(b)

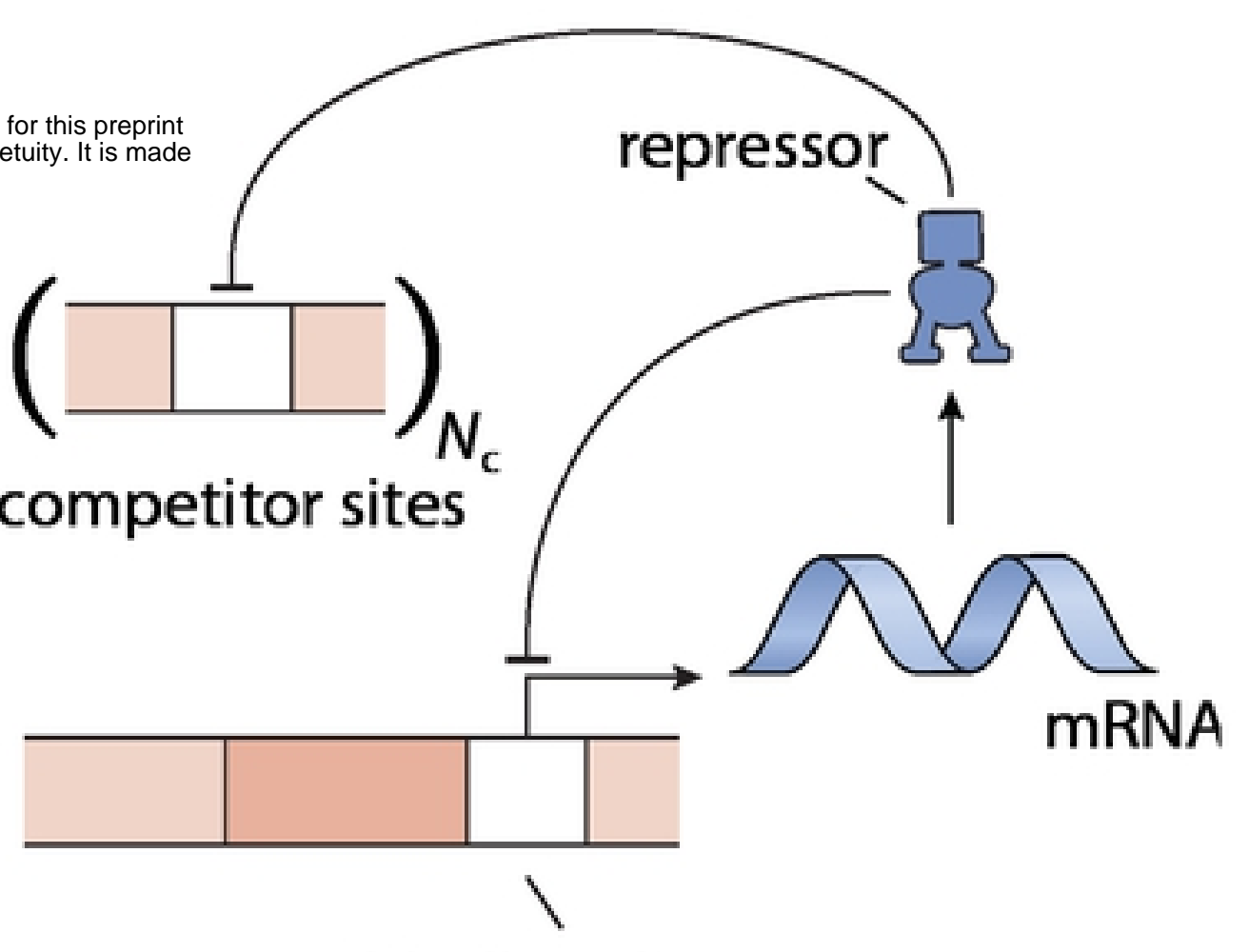

repressor binding site overlapping the promoter

(d)

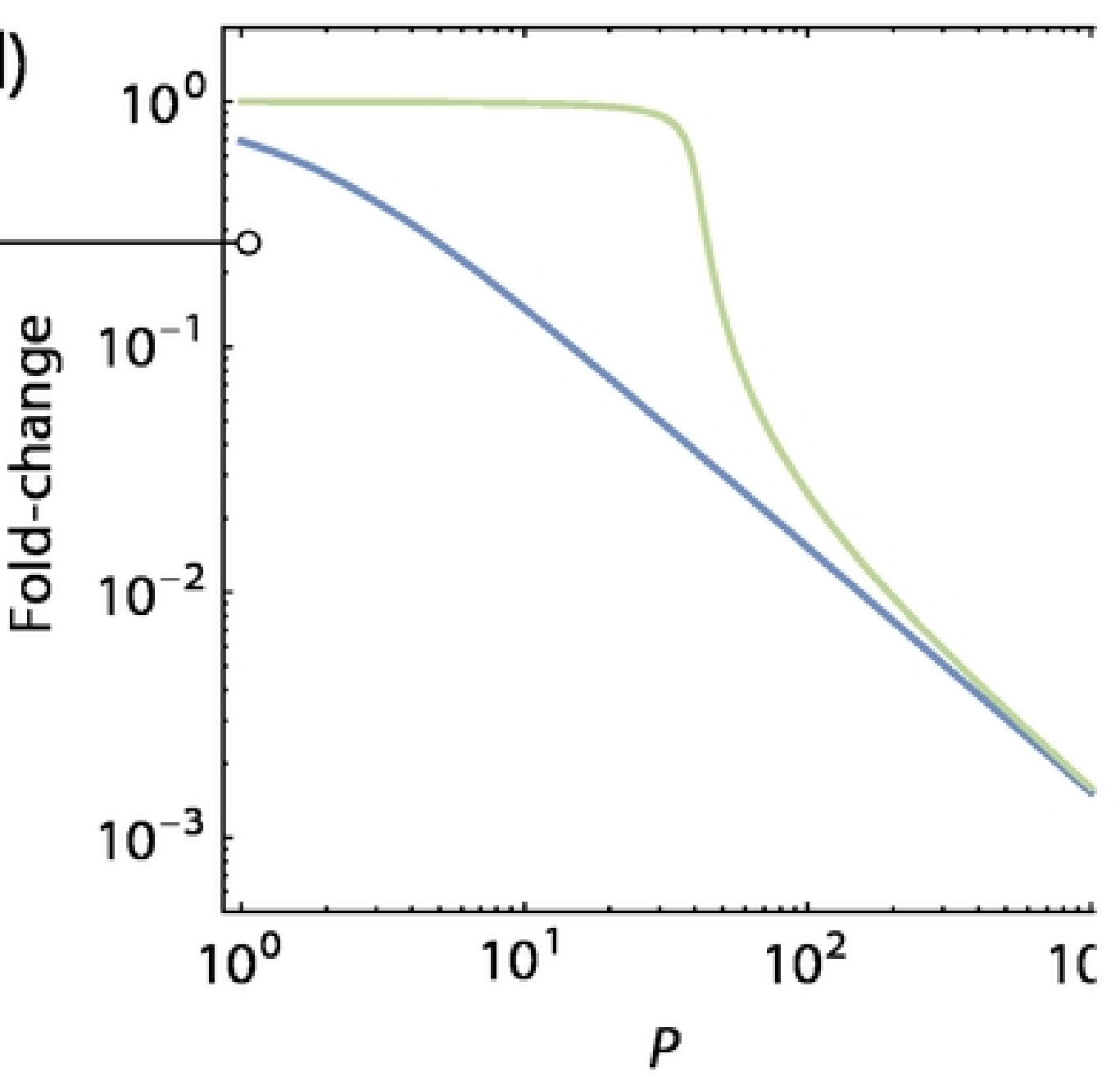

Figure 3 
(a)

mioniv prepint doi: https://doi.org/10.1101/2021.12.01.470700; this version posted Degentber 1, 2021. The copyright holder for this preprint

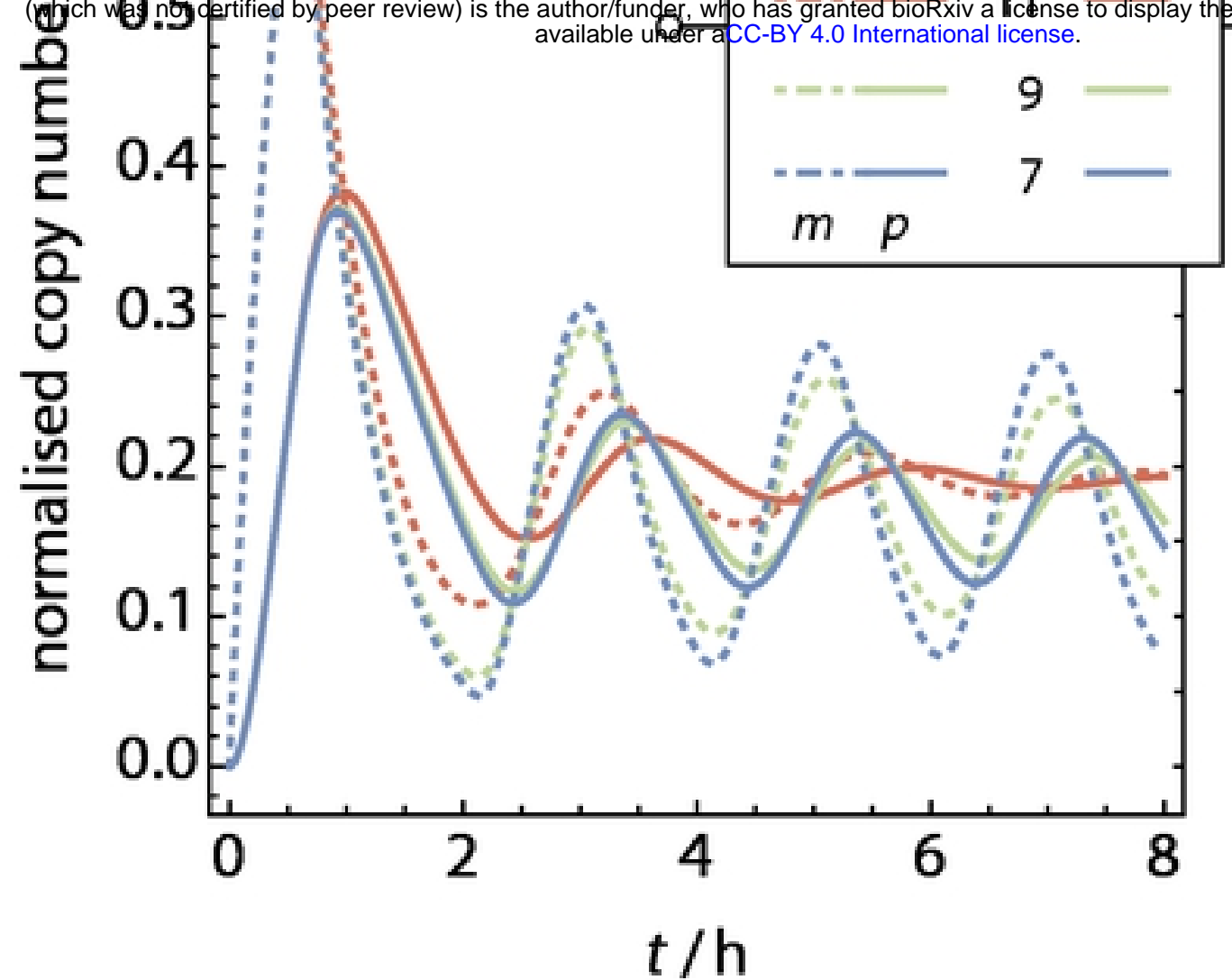

(c)

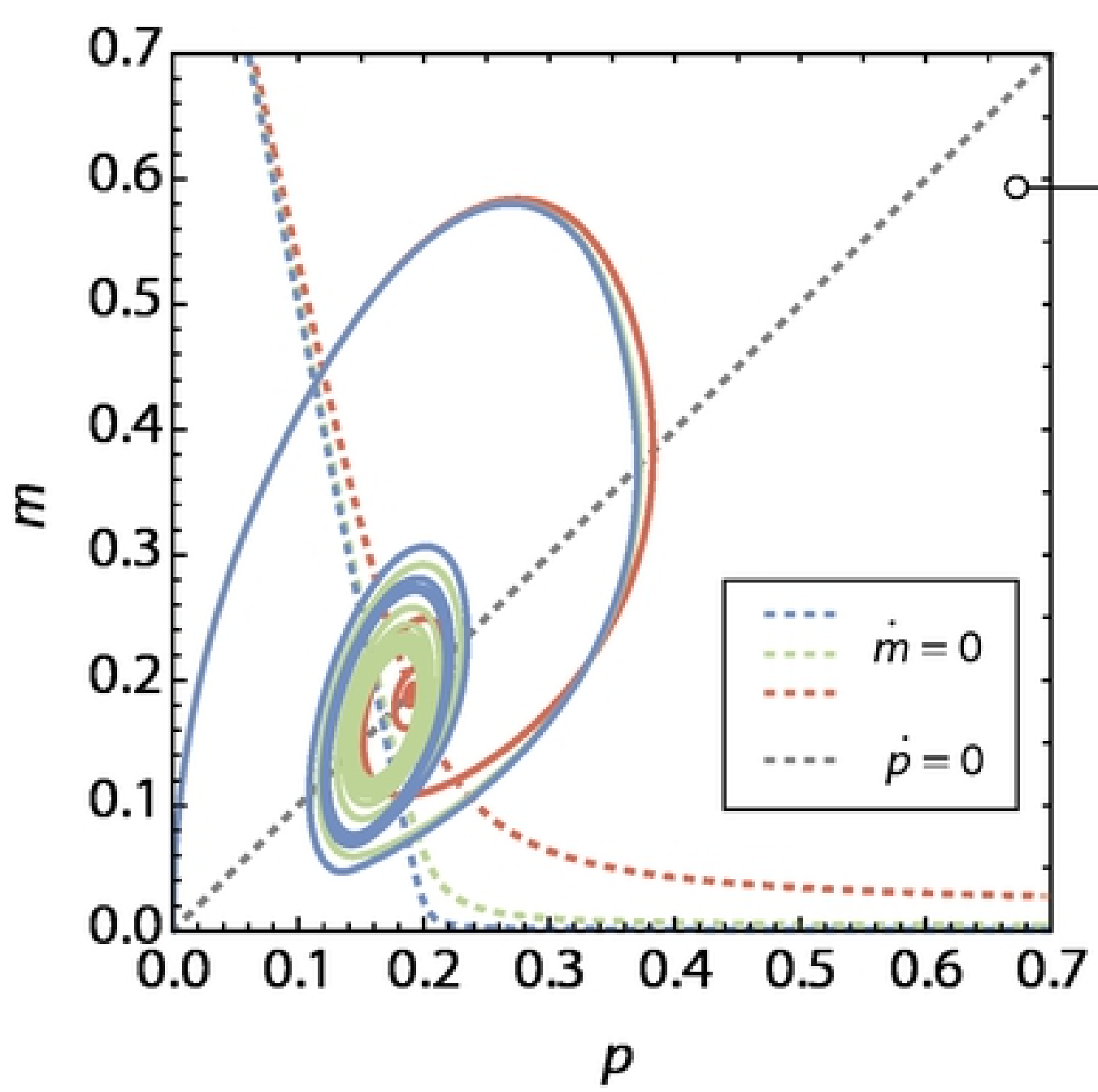

repressor binding site overlapping the promoter repressor

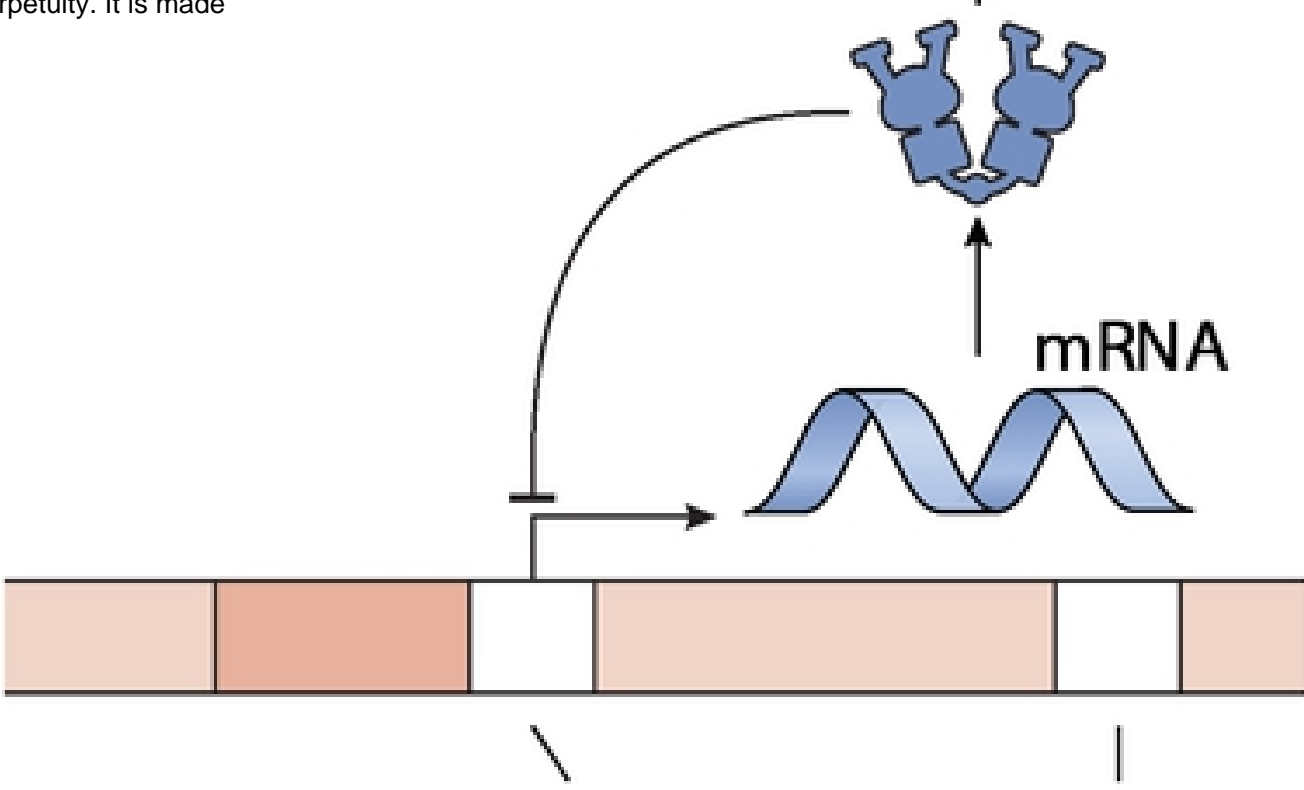

auxiliary operator

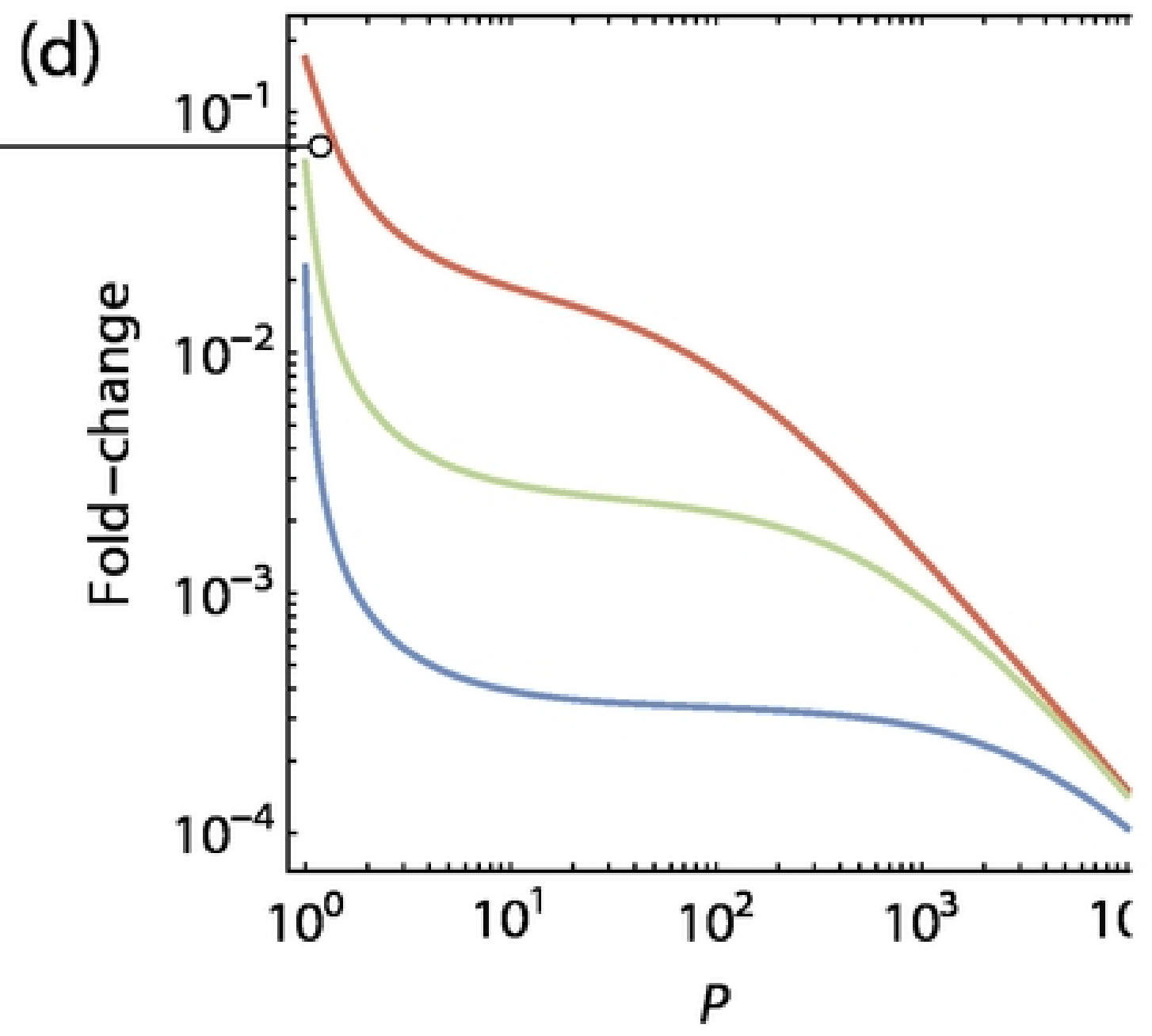

Figure 4 
(a)

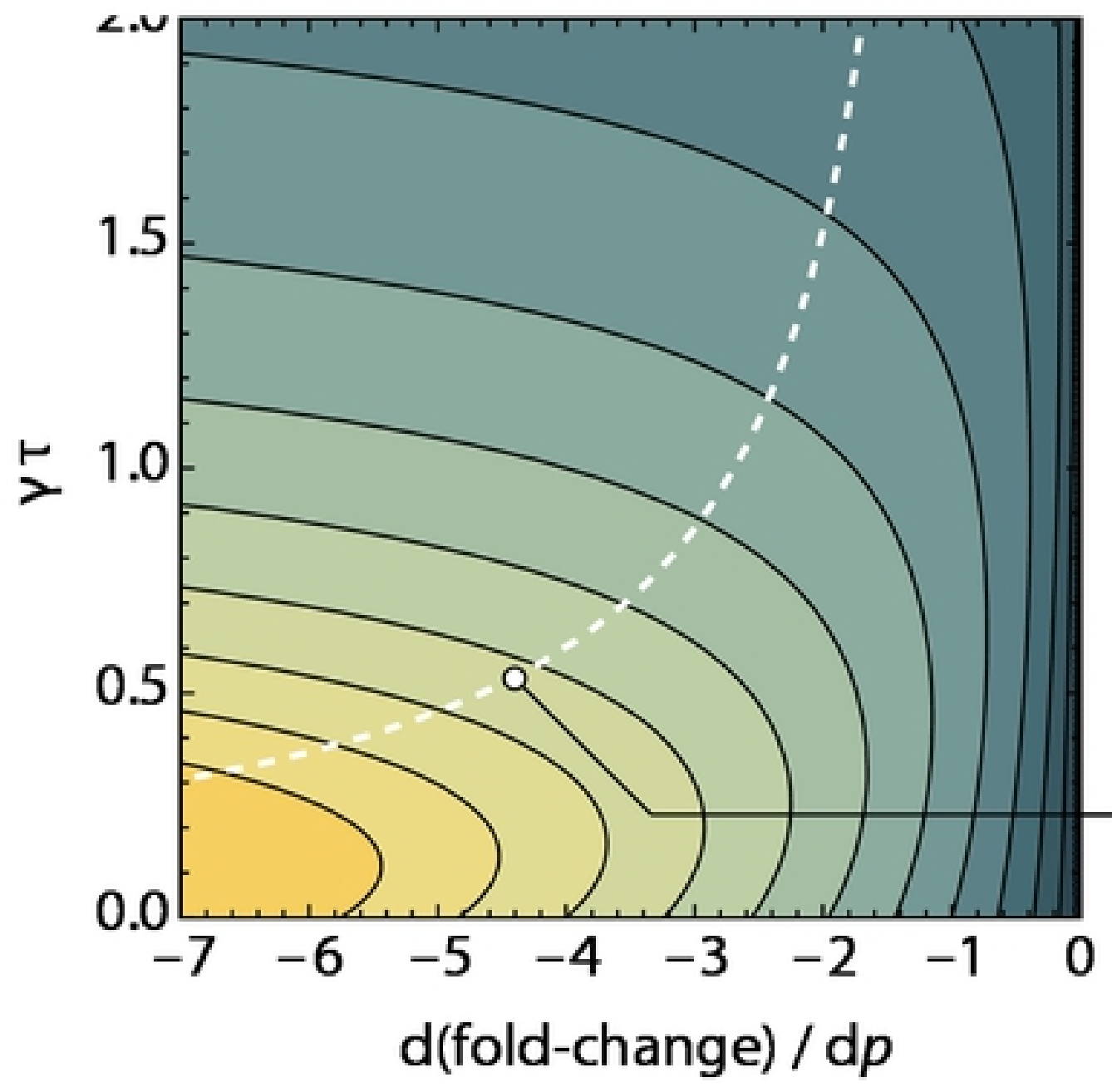

bioRxiv preprint doi: $\begin{array}{llllll}0.4 & 0.8 & 1.2 & 1.6 & 2.0 & 2.4\end{array}$ $\operatorname{Im}\{\lambda\}$

\section{Stability contour}

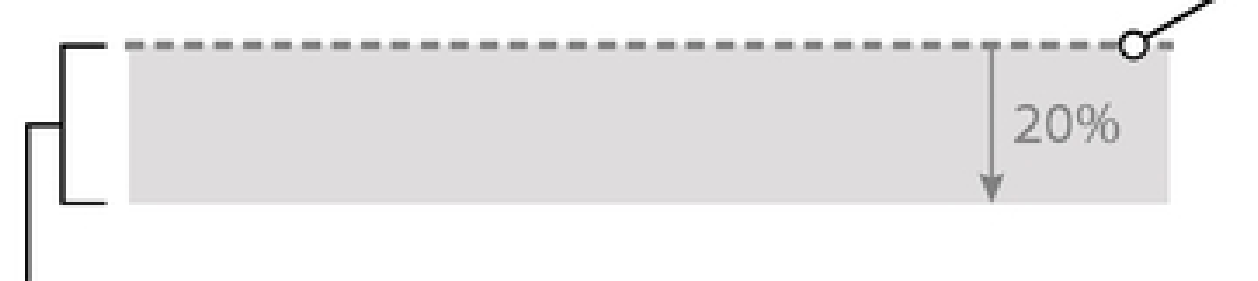

Contour shift at $20 \%$ fluctuations

(b)

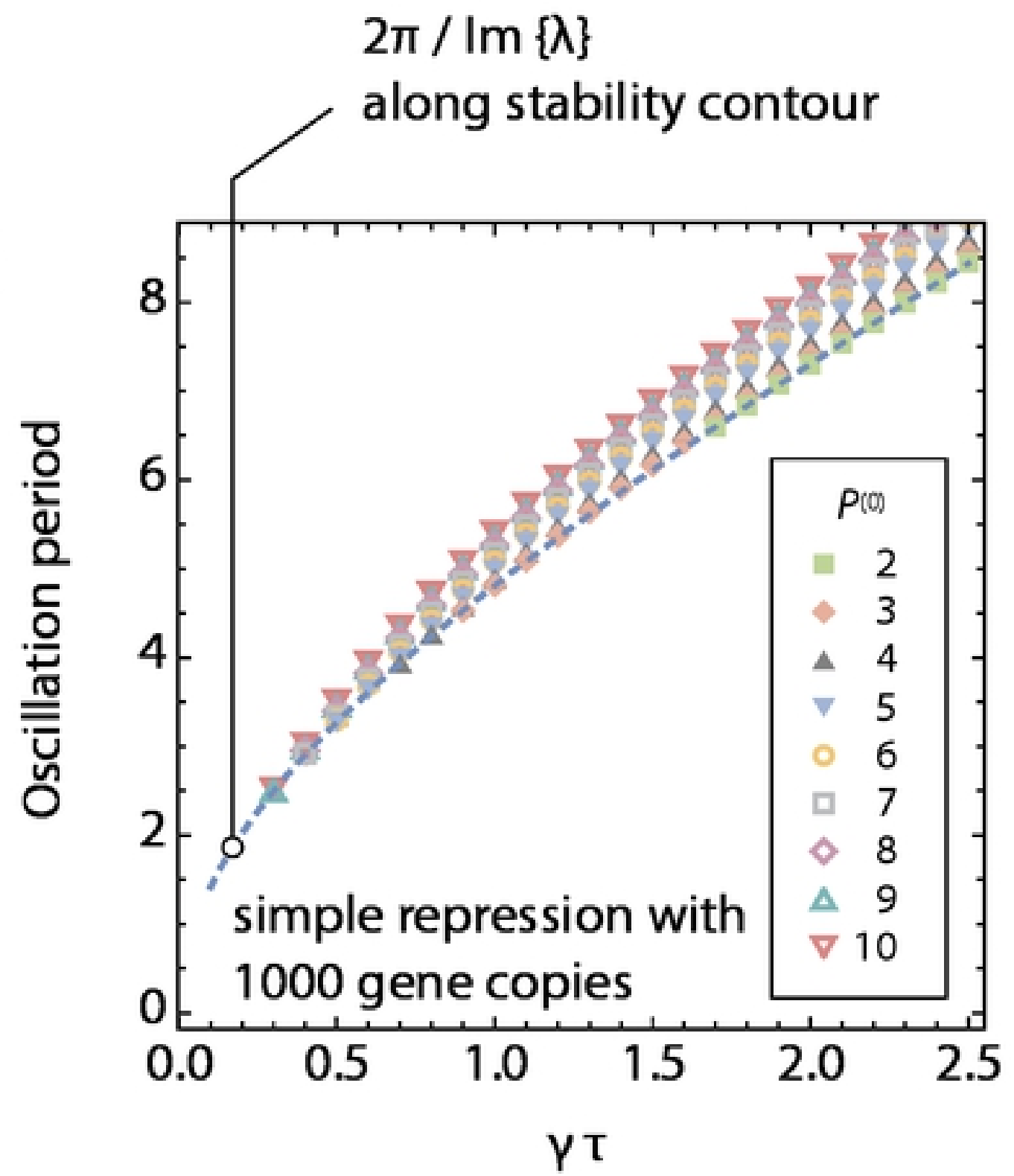

(c)

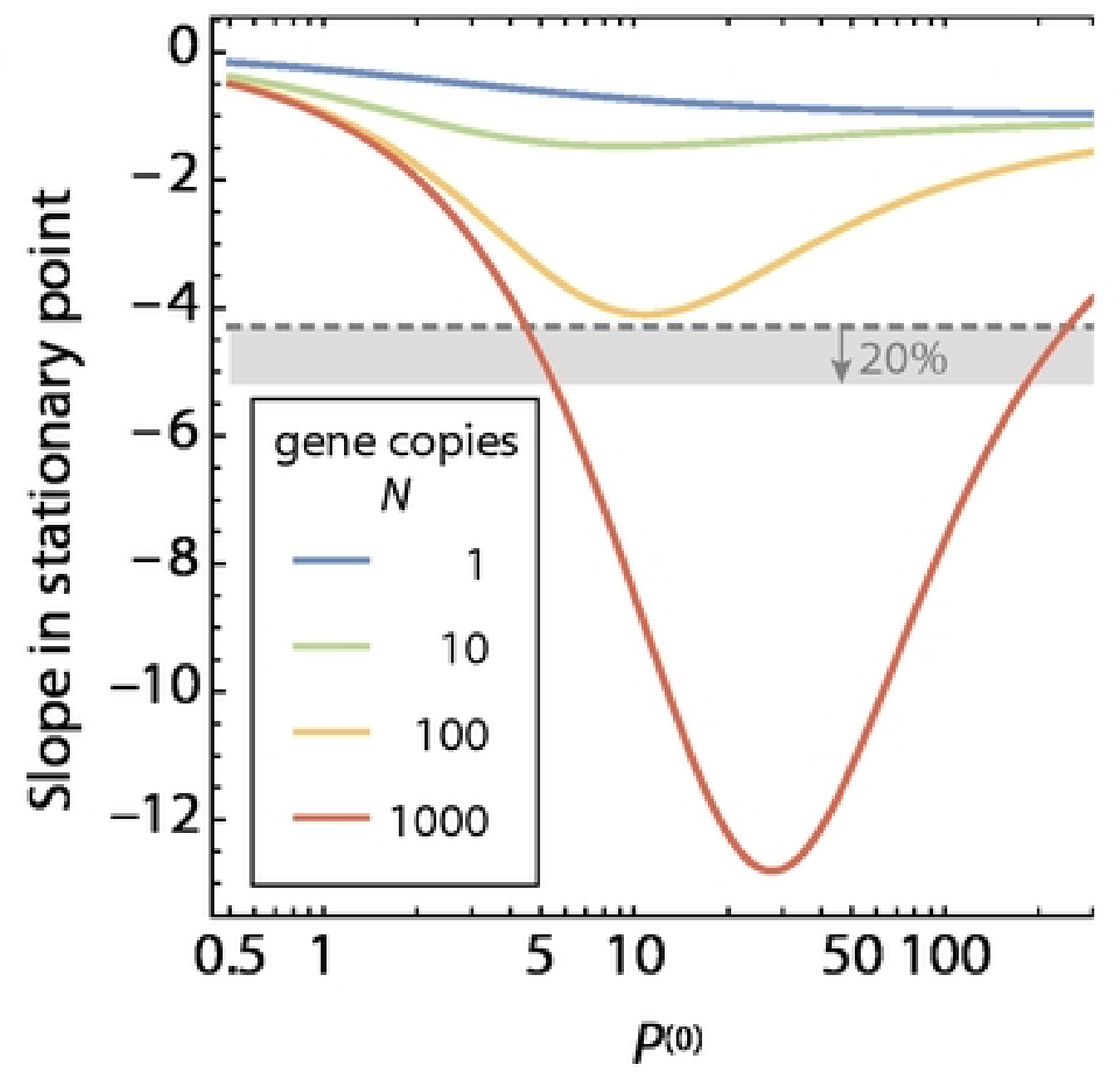

(d)

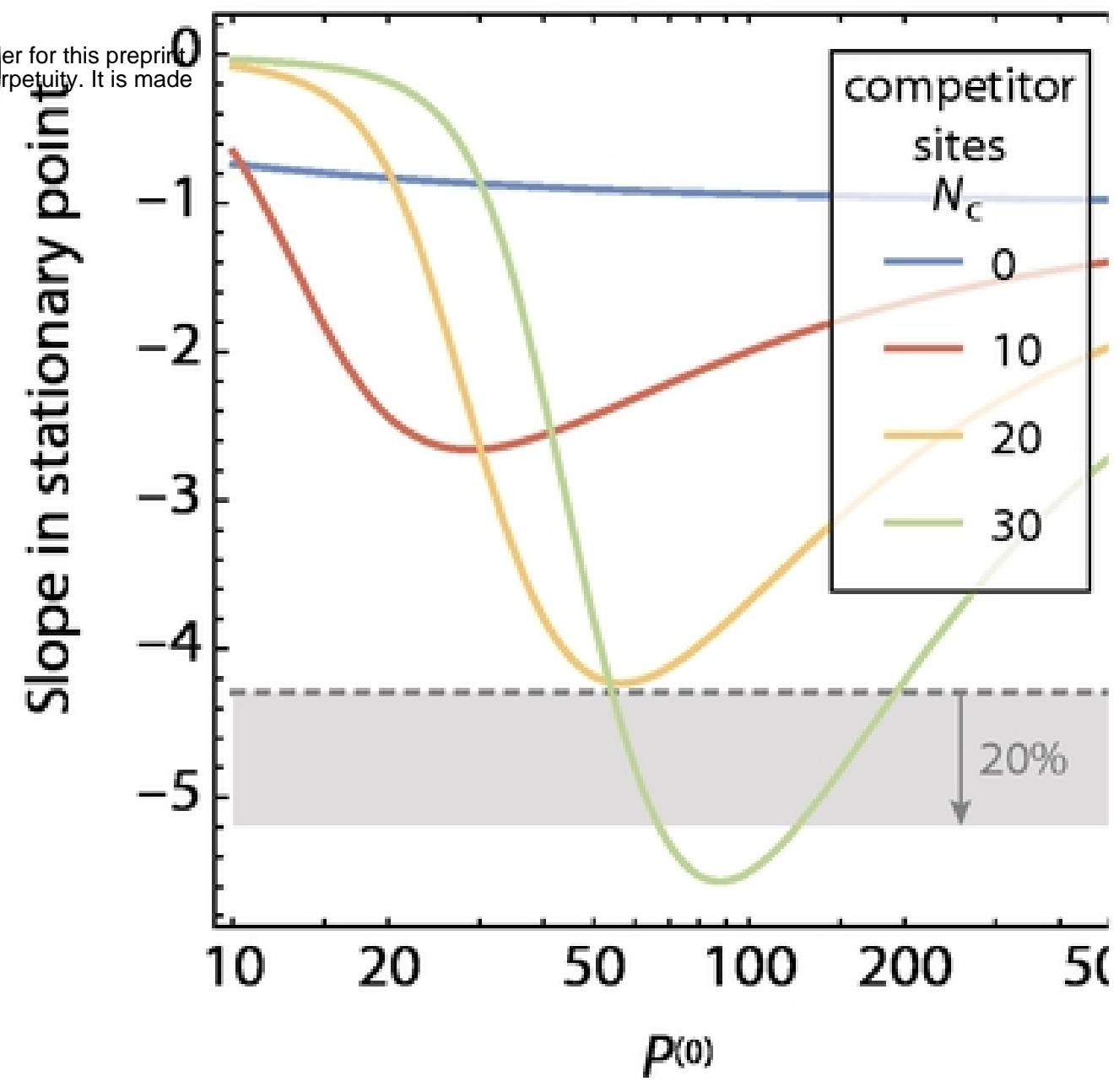

(e)

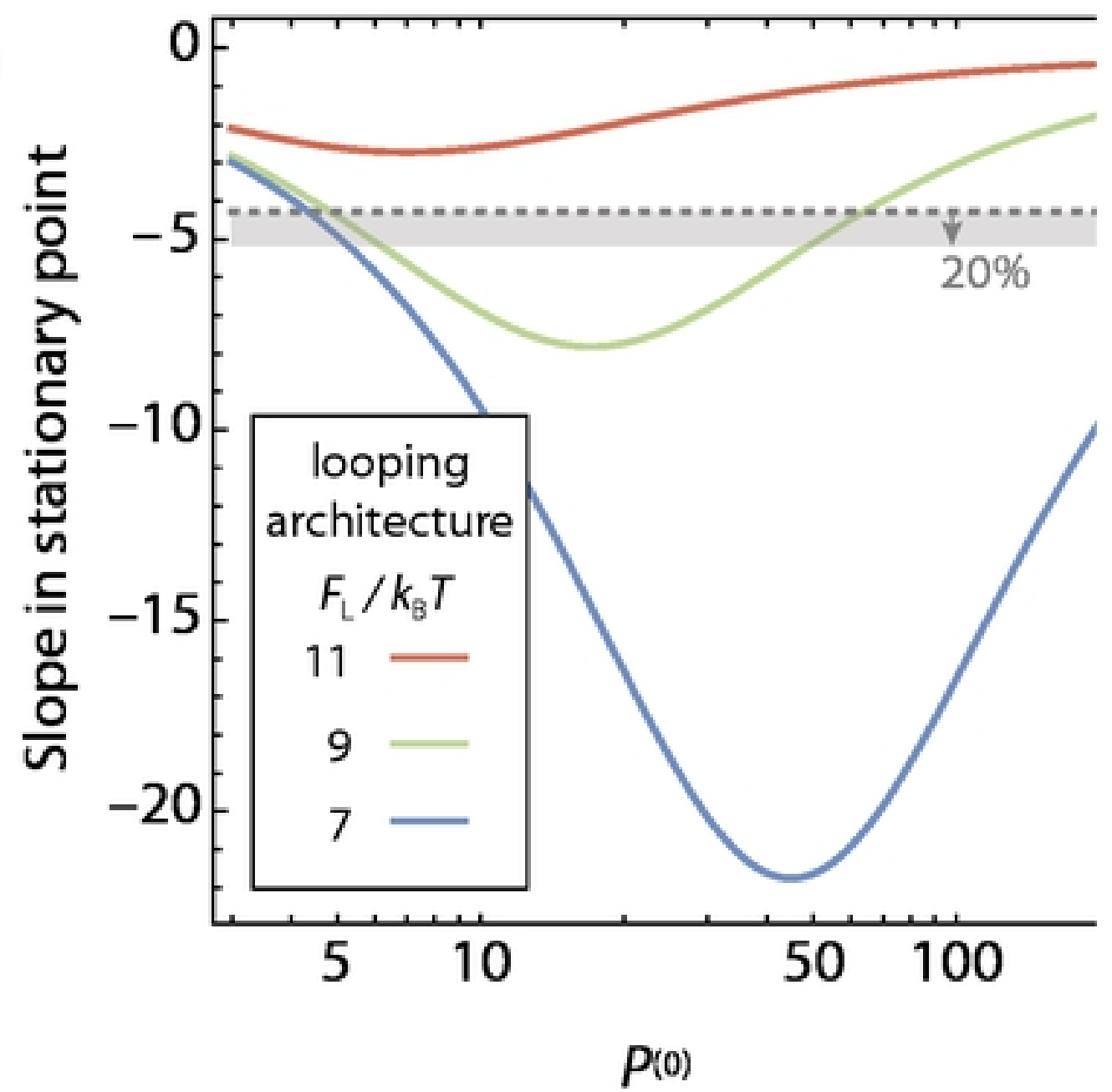

Figure 5 